\title{
1. QUATERNARY, EOCENE, AND CRETACEOUS RADIOLARIANS FROM THE HAWAIIAN ARCH, NORTHERN EQUATORIAL PACIFIC OCEAN ${ }^{1}$
}

\author{
Donna Meyerhoff Hull ${ }^{2}$
}

\begin{abstract}
Deep-sea cores recovered at Sites 842 and 843 on Leg 136 of the Ocean Drilling Program have yielded assemblages of Quaternary, Eocene, and Cretaceous radiolarians from the Hawaiian Arch region of the northern equatorial Pacific Ocean. Reddish-brown clays from Hole 842A (0-9.6 mbsf), Hole 842B ( $0-6.3 \mathrm{mbsf})$, and Hole 843C ( $0-4.2 \mathrm{mbsf})$ contain abundant and diverse assemblages of Quaternary radiolarians consisting of more than 80 species typical of the equatorial Pacific region. Quaternary radiolarians at these sites are assignable to the Quaternary Collosphaera tuberosa Interval Zone and Amphirhopalum ypsilon Interval Zone. The boundary between these zones cannot be determined precisely because of the rarity of zonal markers below surface sediments. Correlations have been made between radiolarian occurrences and magnetostratigraphic events elsewhere in the Pacific Ocean, but similar correlations are difficult at Sites 842 and 843 because of poor subsurface preservation. Chert samples collected from intervals in Cores $842 \mathrm{~B}-10 \mathrm{X}$ and $842 \mathrm{C}-1 \mathrm{~W}$ have yielded radiolarian ages of lower Cenomanian to Santonian and lower Cenomanian, respectively.

Radiolarian assemblages in volcanic sand layers in Sections 6 and 7 of Core 842A-1H (7.5-9.6 mbsf) contain lower and middle Eocene radiolarians admixed with abundant Quaternary faunas. Reworked Eocene radiolarians appear to be restricted to thin layers of volcanic sands within the cores, suggesting deposition by turbidity currents.
\end{abstract}

\section{INTRODUCTION}

Sites $842\left(19^{\circ} 20.18^{\prime} \mathrm{N}, 159^{\circ} 5.33^{\prime} \mathrm{W}\right)$ and $843\left(19^{\circ} 20.53^{\prime} \mathrm{N}\right.$, $159^{\circ} 5.68^{\prime} \mathrm{W}$ ) of Leg 136 of the Ocean Drilling Program were drilled on the Hawaiian Arch, approximately $250 \mathrm{~km}$ southwest of the island of Oahu (Fig. 1). The first objective of this cruise was to drill a hole into the earth's oceanic crust in preparation of a test site for the Ocean Seismographic Network (OSN-1). The goal of this project has been the establishment of a global network of seafloor seismic observatories. The second objective of Leg 136 was to recover sediments and basalt in a lesser known part of the Pacific basin to study: (1) the contamination of Hawaiian magmas by younger sediment; (2) the ages and history of volcanic events in the Hawaiian region; and (3) the age of the basaltic basement.

The sedimentary sequence at both sites consists of approximately $35 \mathrm{~m}$ of reddish-brown deep-sea clays and silty clays overlying nearly $200 \mathrm{~m}$ of claystones and chert. Deep-sea clays in the upper $10 \mathrm{~m}$ in Holes $842 \mathrm{~A}$ and $842 \mathrm{~B}$ and in the upper $4 \mathrm{~m}$ in Hole $843 \mathrm{C}$ contain an abundant and diverse assemblage of Quaternary radiolarians. These faunas are typical of those described previously in the central part of the equatorial Pacific Ocean by Renz (1976) and Johnson (1976), and have similar components to eastern tropical Pacific faunas described by Nigrini (1968). Layers of volcanic sands sampled in Hole 842A also contain a moderately well-preserved, but sparse, assemblage of lower and middle Eocene radiolarians intermixed with the Quaternary faunas. Whereas these assemblages have no biostratigraphic value, the presence of Eocene radiolarians in Quaternary sediments does have implications for deep-sea processes in this region (Riedel and Funnell, 1964; Schreiber, 1969; Garcia, this volume). Cherts from the bottom of Holes $842 \mathrm{~B}$ and $842 \mathrm{C}$ contain a poorly to moderately preserved assemblage of Upper Cretaceous radiolarians. The purpose of this paper is to document the radiolarian assemblages observed from Sites 842 and 843 , and to determine the biostratigraphic ages of the sediments based on those assemblages.

\footnotetext{
'Wilkens, R.H., Firth, J., Bender, J., et al., 1993. Proc. ODP, Sci. Results, 136: College Station, TX (Ocean Drilling Program).

${ }^{2}$ Programs in Geosciences, The University of Texas at Dallas, P.O. Box 830688 , Richardson, TX 75083-0688, U.S.A.
}

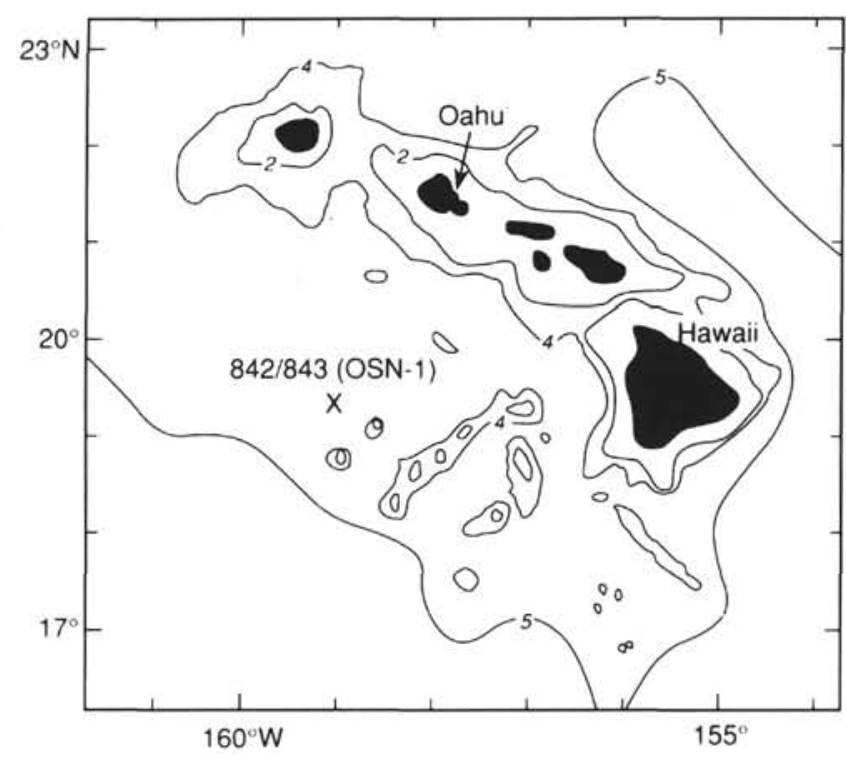

Figure 1. Location of Sites 842 and 843 (OSN-1) on the Hawaiian Arch, 250 $\mathrm{km}$ southwest of Oahu.

\section{SAMPLE AND DATA PREPARATION}

\section{Shipboard Procedures}

Radiolarian-bearing clay samples were disaggregated by boiling in a glass beaker for 2-4 min in a 50:50 mixture of water and hydrogen peroxide. Small amounts of Calgon detergent were added to break up the dense clays. After boiling, each sample was gently washed and sieved through a stack of 60,100 , and 230 mesh $(420,177$, and 63 $\mu \mathrm{m}$, respectively) stainless steel sieves. Residues from each sieve were examined wet; if disaggregation of the clays was incomplete, the residues were returned to the beaker for additional boiling. After completion of this process, the radiolarians were concentrated by a "floating" technique. Cleaned residues with radiolarians were mixed 
with water in a beaker and agitated for $10-15 \mathrm{~s}$ until all of the sediment was in suspension. This mixture was allowed to settle for $20-40 \mathrm{~s}$ until the heavier sediment fell to the bottom of the beaker. Lighter particles, including radiolarian tests, were gently poured off the top and collected in filter paper.

Initial studies aboard ship were conducted with a binocular reflected-light microscope at $70 \times$ to $125 \times$ magnification. Stratigraphically important groups of specimens were picked from the concentrated residue, mounted onto a glass slide with water, dried, then placed in a permanent mounting medium (Norlan Optical Adhesive). These slides were studied under a transmitted light microscope.

\section{Shore-based Procedures}

Samples collected but not processed on board were disaggregated at a shore-based laboratory using the same techniques described above. Specimens for study and documentation were mounted in Hyrax Mounting Medium as opposed to the Norlan Optical Adhesive for better photomicrograph quality.

Cherts collected at Sites 842 and 843 were processed entirely onshore. All of the samples were etched first in hydrochloric acid to determine the presence or absence of calcium carbonate. Leg 136 cherts were almost entirely siliceous, and therefore the hydrofluoric acid (HF) technique of Pessagno and Newport (1972) was used to extract the radiolarians. Accordingly, each sample was placed in a solution of 1 part HF to 9 parts water for approximately $24 \mathrm{hr}$. Each sample was sieved separately after the 24 -hr period, and the residues collected in a stack of 40,80 , and 230 mesh $(425,180,63 \mu \mathrm{m}$, respectively) sieves and dried. Chips and smaller fragments of chert remaining in the 40-mesh sieve were returned to the HF solution for a second 24 -hr period. All of the acid residues were studied under a reflected light microscope.

Eocene and Cretaceous radiolarians are documented in this study using both transmitted-light photography and scanning electron microscope (SEM) photomicrography. Traditionally, Cenozoic assemblages are studied using only transmitted-light techniques, and Cenozoic radiolarian taxonomy relies heavily on knowledge of the internal structure of radiolarian tests. In contrast, a few Quaternary assemblages (e.g., Yamauchi, 1986; Cheng and Yeh, 1989), Paleogene assemblages (e.g., Nishimura, 1986; Suyari and Yamasaki, 1988), and most Mesozoic and Paleozoic assemblages (e.g., Pessagno, 1976, 1977; Cheng, 1986) are studied with reflected-light stereoscopic microscopes and the SEM. The latter techniques are preferred when working with older radiolarians, because their tests are typically replaced by quartz, chalcedony, pyrite, limonite, and other minerals that do not lend themselves well to transmitted-light techniques. An exception to this is a study by Renz (1990), who documented Ordovician radiolarians from Nevada (western North America) using a transmitted-light microscope.

Arguments can be made for and against conducting radiolarian studies with either a transmitted-light microscope or the SEM. Internal structure, easily viewed by transmitted light, is important for proper identification of taxa and for an understanding of evolutionary lineages of many groups of radiolarians at all taxonomic levels. However, extremely detailed and valuable studies of internal cephalic skeletal structure are possible with SEM photomicrography (e.g., Takemura, 1986; Takemura and Yamauchi, 1984). Surficial characteristics such as ridges, striations, and nodes are commonly used in the classification of older faunas, and also may be more promising than shell measurements for paleoecologic applications in younger faunas, such as the Quaternary Botryostrobus auritus/australis "group" (Boltovskoy and Vrba, 1989). Although surficial characteristics are visible in transmitted light by moving the field of focus up and down, the textures and fine details of these features can be studied more accurately with the high degree of magnification and resolution made possible by an SEM. A study by Caulet and Nigrini (1988) of the Neogene genus Pterocorys utilizes both methods to successfully illustrate both internal and exter- nal characteristics of this genus that are taxonomically significant. Thus, as noted by other authors (Pessagno et al., 1984, 1986), the most comprehensive approach is to present both SEM and transmitted light photomicrographs, recognizing that to do so requires added time and effort. Reworked Eocene specimens included herein contain dense clays within the tests that cannot be removed without risk of losing these rarer specimens. These radiolarians and quartz-replaced Cretaceous species are illustrated with SEM photomicrography. Quaternary and better-preserved Eocene species are documented with transmittedlight photography.

\section{Abundance and Preservation}

Relative abundance and preservation of Quaternary radiolarians at Sites 842 and 843 are shown in Table 1. Abundances are estimated by counting the number of specimens observed traversing a $9 \times 8 \mathrm{~cm}$ tray containing approximately $1 \mathrm{~g}$ of residue. The abundances are recorded as follows:

$$
\begin{aligned}
& R=\text { rare }(1-49 \text { specimens per tray }), \\
& F=\text { few }(50-99 \text { specimens per tray }), \\
& C=\text { common }(100-200 \text { specimens per tray }), \text { and } \\
& A=\text { abundant }(>200 \text { specimens per tray }) .
\end{aligned}
$$

Concentrations are relatively high in these samples, because of the laboratory technique of concentrating the radiolarians in the residues before drying. Species abundances are recorded on Table 1 as follows:

$$
\begin{aligned}
& \mathrm{R}=\text { rare }(1-2 \text { specimens }), \\
& \mathrm{F}=\text { few }(3-5 \text { specimens }), \\
& \mathrm{C}=\text { common }(6-20 \text { specimens }), \text { and } \\
& \mathrm{A}=\text { abundant }(>20 \text { specimens }) .
\end{aligned}
$$

The state of preservation of radiolarian assemblages is documented as follows (Table 1):

$\mathrm{P}=$ poor: radiolarians completely or nearly completely covered/infilled with clay; pore structure largely obscured.

$\mathrm{M}=$ moderate: some clay in pores of radiolarians; overall structure still visible.

$\mathrm{G}$ = good: radiolarians free of clay, pore structures and ornamentation easily visible.

Many samples possess a wide range in the quality of preservation. This may be due in part to increasing dissolution of more fragile forms with depth, but varying preservation is also caused by the thorough infilling and difficult removal of clays within many specimens, particularly spongy forms. Also, many of the species documented in this study have long stratigraphic ranges, and their poor preservation may be a reflection of the reworking of older specimens.

\section{BIOSTRATIGRAPHY}

Over the last 25 years, numerous studies have documented Cenozoic radiolarian faunas from various parts of the equatorial Pacific Ocean. Among the most significant of these taxonomic reports are Nigrini (1968, 1971), Riedel and Sanfilippo (1971, 1978), Renz (1976), Dinkelman (1973), and Johnson (1976). Many groups of Paleocene through Quaternary radiolarians have been well described and documented, and biostratigraphic zonation schemes have been established for different regions of the Pacific Ocean (e.g., Nigrini, 1971; Riedel and Sanfilippo, 1978; Goll, 1980). There are still, however, several groups of abundant and diverse Cenozoic radiolarians whose classification is confusing, at best (e.g., the genera Theocalyptra and Clathrocyclas). These groups and others are in need of major revision, and their stratigraphic utility remains partly obscure until such comprehensive studies are completed. 


\section{Quaternary Radiolarian Biostratigraphy}

Age determinations and zonal assignments for Sites 842 and 843 have been derived from Nigrini (1971), who established the first Quaternary radiolarian zonation for the equatorial Pacific Ocean. Modifications to this zonation were later proposed by Johnson and Knoll (1975). Detailed radiolarian biostratigraphy for the Quaternary has been difficult to achieve due to the short span of time involved and the presence of few rapidly evolving taxa during this time. Furthermore, some of the taxa showing speciation during the Quaternary possess fragile, thin tests, that are susceptible to both chemical dissolution and physical destruction. Nevertheless, radiolarian "events" have occurred throughout the Quaternary, and several of these have been used to subdivide this epoch into four radiolarian biozones: Buccinosphaera invaginata Range-Zone, Collosphaera tuberosa Interval-Zone, Amphirhopalum ypsilon Interval-Zone and Anthocyrtidium angulare Interval-Zone (Nigrini, 1971; Johnson and Knoll, 1975; Sanfilippo et al., 1985, p. 645). Johnson et al. (1989) subdivided the Collosphaera tuberosa Interval-Zone into the Collosphaera tuberosa Zone and the Stylatractus universus Zone on the basis of data collected in the Central Indian Basin and Ninetyeast Ridge in the Indian Ocean. Inasmuch as there are taxonomic differences between the Indian Ocean and Pacific Ocean Quaternary faunas, this author has maintained the use of $\mathrm{Col}$ losphaera tuberosa Interval-Zone as defined for the Pacific region (sensu Johnson and Knoll, 1975, Sanfilippo et al., 1985).

Two radiolarian zones, the Collosphaera tuberosa Interval-Zone and the Amphirhopalum ypsilon Interval-Zone, are present in cores at Sites 842 and 843 . The top of the Collosphaera tuberosa IntervalZone is recognized as the first morphotypic appearance of Buccinosphaera invaginata, which undergoes a transition from Collosphaera orthoconus (= Collosphaera sp. A of Johnson and Knoll, $1975)$ to B. invaginata at this stratigraphic level. The base of the Collosphaera tuberosa Interval-Zone is defined by the first occurrence of $C$. tuberosa. The top of the Amphirhopalum ypsilon Interval-Zone is directly below the first occurrence of $C$. tuberosa. Absolute ages for Neogene radiolarian events in the central Pacific Ocean, based on correlations between these events and magnetostratigraphy, are from Johnson and Knoll (1975), with recalibrations to the 1992 paleomagnetic time scale provided by Garcia (pers. comm., 1993).

\section{Site 842}

\section{Hole $842 A$}

Core $842 \mathrm{~A}-1 \mathrm{H}$ recovered $9.66 \mathrm{~m}$ of reddish-brown clay rich in Quaternary radiolarians (Dziewonski, Wilkens, Firth, et al., 1992). Sample 842A-1H-1, 28-30 cm, contains a well-preserved radiolarian fauna consisting of more than 60 Quaternary species (Table 1), including Collosphaera orthoconus. Johnson and Knoll (1975) estimated the first appearance of Collosphaera orthoconus at $0.61 \mathrm{Ma}$, and placed its evolutionary transition to Buccinosphaera invaginata at $0.21 \pm 0.02 \mathrm{Ma}$. Recalibration to the new paleomagnetic time scale (1992) gives an age of $0.24 \pm 0.004 \mathrm{Ma}$ for this transition (Garcia, pers. comm., 1993). Johnson et al. (1989) placed this transition at 0.17 $\mathrm{Ma}$ on the basis of correlations between radiolarian and magnetostratigraphic events in the Indian Ocean. If Johnson and Knoll's (1975) evolutionary scheme for $B$. invaginata is correct, the specimens figured herein (Pl. 1, Figs. 18, 19) appear to be "late" forms (i.e., very close to the transition into $B$. invaginata). Therefore, the presence of a late form of Collosphaera orthoconus indicates that surface sediments at Hole $842 \mathrm{~A}$ are assignable to the upper part of the Collosphaera tuberosa Interval-Zone (Fig. 2).

Another Quaternary marker species, Axoprunum angelinum, is present at and below 1.29-1.31 mbsf (Sample 842A-1H-1, 129-131 $\mathrm{cm})$. Overall, this species is rare in its occurrence in both Holes $842 \mathrm{~A}$ and 842B. Johnson and Knoll (1975) placed the final occurrence of A. angelinum in the equatorial Pacific Ocean at $0.32 \mathrm{Ma}(=0.36 \pm 0.02$
Ma by the 1992 paleomagnetic scale; Garcia, pers. comm., 1993), in approximately the middle of the Collosphaera tuberosa IntervalZone. Morley and Shackleton (1978) place the global final occurrence of this species (=Stylatractus universus therein) at $0.42 \mathrm{Ma}$. It should be noted, however, that this is a relatively robust form that may be capable of withstanding reworking. Thus, either the sediments below 1.3 mbsf are older than $0.32-0.42 \mathrm{Ma}$, or, possibly, A. angelinum has been reworked into slightly younger sediments.

Collosphaera tuberosa is absent in Hole $842 \mathrm{~A}$, although it has been recovered from surface sediments at Hole $842 \mathrm{~B}$ ( 10 m east of $842 \mathrm{~A})$. In the absence of marker taxa, the boundary between the Collosphaera tuberosa Interval-Zone and the underlying Amphirhopalum ypsilon Interval-Zone can only be approximated (Fig. 2). According to the magnetostratigraphy (Helsley, pers. comm., 1992), the boundary between the Brunhes and Matuyama Epochs is at $1.96 \mathrm{mbsf}$, and the Jaramillo Event occurs between 4.42 and 4.97 mbsf. Correlation between radiolarian biostratigraphic events and paleomagnetic events by several workers (Hays and Berggren, 1971; Johnson and Knoll, 1975) at other localities in the Pacific Ocean place the Brunhes/ Matuyama boundary near the middle of the Amphirhopalum ypsilon Interval-Zone and the Jaramillo Event within the lower part of the Amphirhopalum ypsilon Interval-Zone. Because zonal markers are rare in Holes $842 \mathrm{~A}$ and $842 \mathrm{~B}$, these correlations cannot be confirmed. Furthermore, Sample $842 \mathrm{~A}-1 \mathrm{H}-6,130 \mathrm{~cm}$ ( $8.8 \mathrm{mbsf}$ ), yielded nannofossils assignable to the lower part of Quaternary Zone NN19 (early Pleistocene), and correlates with an absolute age older than or equal to 1.3 Ma (Dziewonski, Wilkens, Firth, et al., 1992). Radiolarians are present in samples above and below this nannofossil-rich interval, but no marker taxa for the early Pleistocene Anthocyrtidium angulare Interval-Zone have been found to confirm this age.

\section{Hole $842 B$}

The first core recovered from Hole $842 \mathrm{~B}, 10$ m east of Hole $842 \mathrm{~A}$, contains a rich Quaternary radiolarian fauna, nearly identical in composition to samples collected from Hole 842A (Table 1). Surface sediments in Sample 842B-1H-1, 9-11 cm, include Collosphaera orthoconus, Collosphaera tuberosa, and Axoprunum angelinum. As in Hole $842 \mathrm{~A}$, the presence of Collosphaera orthoconus, prior to its evolutionary transition into Buccinosphaera invaginata, places this radiolarian assemblage in the Collosphaera tuberosa Interval-Zone. Recalibrating Johnson and Knoll's (1975) absolute ages for Quaternary radiolarian events in the equatorial Pacific Ocean, this sample is younger than the first appearance of Collosphaera tuberosa at $0.42 \pm$ $0.01 \mathrm{Ma}$, and older than the final occurrence of Collosphaera orthoconus at $0.24 \pm 0.004 \mathrm{Ma}$ (Fig. 2). It should be noted that Johnson et al. (1989) documented the first appearance datum of C. tuberosa at a slightly older age of $0.5 \mathrm{Ma}$ in the Indian Ocean. The final appearance of $A$. angelinum, a single occurrence in surface sediments of Hole $842 \mathrm{~B}$, may be used to further constrain the age, but it is also possible that this specimen is reworked.

As in Hole 842A, the boundary between the Collosphaera tuberosa Interval-Zone and the Amphirhopalum ypsilon Interval-Zone cannot be determined precisely. Collosphaera tuberosa is present only in the surface sediments of Sample 842B-1H-1, 9-11 cm. Its first stratigraphic occurrence in Hole $842 \mathrm{~B}$, which marks the base of the $C$. tuberosa Interval-Zone, is unknown. The Brunhes/Matuyama boundary has been placed at $1.8 \mathrm{mbsf}$, and the Jaramillo Event between 3.92 and 4.4 mbsf (Fig. 2; Helsley, pers. comm., 1992). Radiolarian assemblages in the basal section of Core 842B-1H are assigned to the Amphirhopalum ypsilon Interval-Zone; marker taxa indicative of the underlying Anthocyrtidium angulare Interval-Zone are absent. Fourteen samples collected from Core 842B-2H and the upper part of Core 842B-3H are barren of all microfossils, including radiolarians. The remainder of Core $842 \mathrm{~B}-3 \mathrm{H}$ and Cores $842 \mathrm{~B}-4 \mathrm{H}$ and $842 \mathrm{~B}-5 \mathrm{H}$ contained upper Oligocene?/early Miocene ichthyoliths (Firth and Hull, this volume). 
Table 1. Estimated abundance and preservation of selected Quaternary radiolarian species from Holes $842 \mathrm{~A}, 842 \mathrm{~B}$, and $843 \mathrm{C}$.

\begin{tabular}{|c|c|c|c|c|c|c|c|c|c|c|c|c|c|c|c|c|c|c|c|c|c|c|}
\hline $\begin{array}{l}\text { Site, section, } \\
\text { core }\end{array}$ & Interval (cm) & $\begin{array}{l}\text { Abundance/ } \\
\text { Preservation }\end{array}$ & 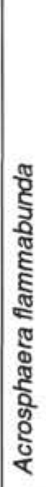 & $\begin{array}{l}\pi \\
\frac{\pi}{0} \\
\frac{\pi}{2} \\
\frac{\pi}{2} \\
\frac{\pi}{0} \\
\frac{\pi}{0} \\
\frac{\pi}{0} \\
\frac{0}{0} \\
\frac{\pi}{4}\end{array}$ & 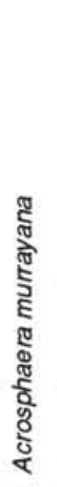 & 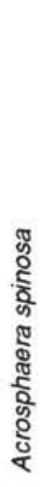 & 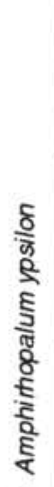 & 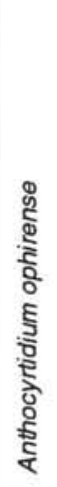 & 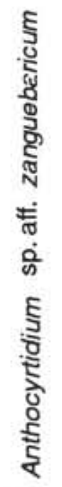 & 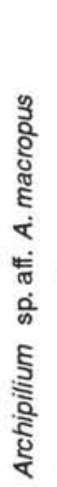 & 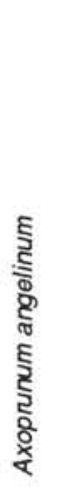 & 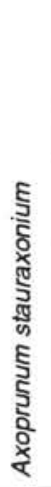 & 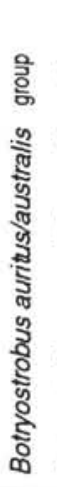 & 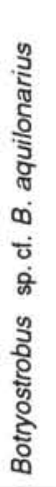 & 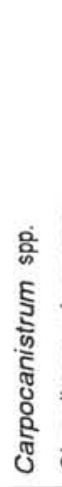 & 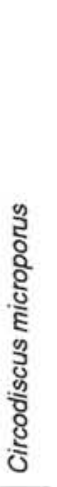 & $\begin{array}{l}\text { के } \\
\text { है } \\
\text { तु } \\
8 \\
\frac{\pi}{5} \\
\frac{\pi}{0} \\
0\end{array}$ & 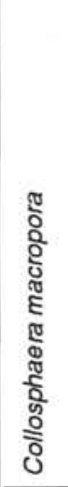 & 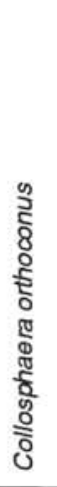 & 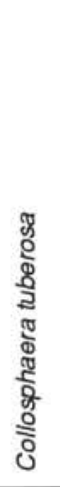 & $\begin{array}{l}\frac{\pi}{0} \\
5 \\
\frac{5}{0} \\
\frac{0}{0} \\
\frac{\pi}{0} \\
\frac{0}{5} \\
\text { है } \\
0\end{array}$ & 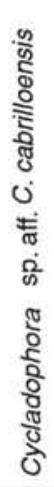 \\
\hline $842 \mathrm{~A}-1 \mathrm{H}-1$ & $28-30$ & $A / M-G$ & R & $\mathrm{F}$ & & A & A & A & $\mathrm{R}$ & $\mathrm{R}$ & & & $\mathrm{F}$ & $\mathrm{R}$ & $\mathrm{C}$ & $\mathrm{F}$ & & $\mathrm{F}$ & $\mathrm{F}$ & & A & $\mathrm{R}$ \\
\hline $842 \mathrm{~A}-1 \mathrm{H}-1$ & $129-131$ & $A / M-G$ & & $\mathrm{R}$ & & $R$ & A & $\mathrm{C}$ & & $\mathrm{R}$ & R & & $\mathrm{F}$ & & A & $\mathrm{R}$ & & & & & C & \\
\hline $842 \mathrm{~A}-1 \mathrm{H}-2$ & $130-132$ & $A / M-G$ & & $\mathrm{R}$ & & C & C & C & & & & $\mathrm{F}$ & $\mathrm{F}$ & & $R$ & $\mathrm{C}$ & & R & & & C & \\
\hline $842 \mathrm{~A}-1 \mathrm{H}-3$ & $7-9$ & $A / M-G$ & $R$ & $\mathrm{~F}$ & & $\mathrm{~F}$ & C & C & & $R$ & & $\mathrm{~F}$ & & & C & $\mathrm{C}$ & & $\mathrm{R}$ & & & $C$ & \\
\hline $842 \mathrm{~A}-1 \mathrm{H}-3$ & $129-131$ & ANM-G & R & C & & C & C & C & & & & $\mathrm{A}$ & $C$ & & C & & & & & & C & $R$ \\
\hline $842 \mathrm{~A}-1 \mathrm{H}-4$ & $129-131$ & $\mathrm{C} / \mathrm{M}$ & & & & & C & C & & & $\mathrm{R}$ & $\mathrm{R}$ & $\mathrm{R}$ & & $\mathrm{C}$ & $\mathrm{R}$ & & & & & C & \\
\hline $842 \mathrm{~A}-1 \mathrm{H}-5$ & $8-10$ & C/P-G & & & & $\mathrm{R}$ & C & & & & & $\mathrm{A}$ & & & & $\mathrm{R}$ & & & & & $\mathrm{C}$ & \\
\hline $842 \mathrm{~A}-1 \mathrm{H}-5$ & $129-131$ & C/P-G & & $\mathrm{R}$ & & $\mathrm{F}$ & C & & & & & $\mathrm{A}$ & & & & & & & & & $\mathrm{C}$ & \\
\hline $842 \mathrm{~A}-1 \mathrm{H}-6$ & $128-132$ & $A / P-M$ & & & & $\mathrm{~F}$ & C & $\mathrm{F}$ & & & $R$ & C & & & C & & & & & & C & \\
\hline $842 \mathrm{~A}-1 \mathrm{H}-7$ & $9-11$ & A/P-M & & & & & C & & & & & $\mathrm{F}$ & $\mathrm{F}$ & & $R$ & $\mathrm{~F}$ & & & & & $\mathrm{C}$ & \\
\hline $842 \mathrm{~B}-1 \mathrm{H}-1$ & $9-11$ & A/M-G & $\mathrm{A}$ & $\mathrm{R}$ & $R$ & $\mathrm{~F}$ & $C$ & C & $\mathrm{R}$ & & $\mathrm{R}$ & $\mathrm{A}$ & $C$ & $R$ & $\mathrm{~F}$ & $R$ & & $R$ & $\mathrm{R}$ & $R$ & $\mathrm{C}$ & \\
\hline $842 \mathrm{~B}-1 \mathrm{H}-1$ & $126-128$ & C/P-G & $\mathrm{R}$ & $\mathrm{R}$ & & A & A & $\mathrm{R}$ & & & $\mathrm{R}$ & $\mathrm{F}$ & $R$ & & $\mathrm{~F}$ & & & $R$ & & & $\mathrm{~F}$ & \\
\hline $842 \mathrm{~B}-1 \mathrm{H}-2$ & $7-9$ & $\mathrm{R} / \mathrm{P}$ & & & & & & & & & & & & & & & & & & & & \\
\hline $842 \mathrm{~B}-1 \mathrm{H}-2$ & $126-128$ & A/P-G & & & & $\mathrm{F}$ & A & $\mathrm{C}$ & & R & & $\mathrm{R}$ & & & $R$ & $R$ & $R$ & $R$ & & & $\mathrm{R}$ & $\mathrm{R}$ \\
\hline $842 \mathrm{~B}-1 \mathrm{H}-3$ & $9-11$ & AVP-G & A & $R$ & & $\mathrm{~F}$ & C & $\mathrm{R}$ & & $R$ & & $\mathrm{R}$ & $R$ & & $\mathrm{R}$ & & & & & & $\mathrm{F}$ & $\mathrm{R}$ \\
\hline $842 \mathrm{~B}-1 \mathrm{H}-3$ & $126-128$ & A/M-G & $\mathrm{R}$ & & & $\mathrm{R}$ & $\mathrm{F}$ & $\mathrm{R}$ & & & & & $R$ & & & & & & & & $R$ & \\
\hline $842 \mathrm{~B}-1 \mathrm{H}-4$ & $9-11$ & Barren & & & & & & & & & & & & & & & & & & & & \\
\hline $842 \mathrm{~B}-1 \mathrm{H}-4$ & $126-128$ & $\mathrm{C} / \mathrm{P}-\mathrm{M}$ & & & & $\mathrm{R}$ & $\mathrm{F}$ & & & & & & $\mathrm{F}$ & & $R$ & & & & & & $\mathrm{R}$ & \\
\hline $842 \mathrm{~B}-1 \mathrm{H}-5$ & $8-10$ & $\mathrm{C} / \mathrm{M}-\mathrm{G}$ & $\mathrm{R}$ & & & $\mathrm{F}$ & $\mathrm{F}$ & $\mathrm{R}$ & & $\mathrm{F}$ & & $\mathrm{R}$ & $\mathrm{R}$ & & $\mathrm{F}$ & & & & & & C & \\
\hline $843 \mathrm{C}-1 \mathrm{H}-1$ & $34-36$ & $A / G$ & & $\mathrm{R}$ & & $\mathrm{C}$ & $\mathrm{F}$ & $\mathrm{F}$ & & R & & $\mathrm{F}$ & $R$ & $\mathrm{R}$ & $\mathrm{C}$ & $\mathrm{R}$ & & $\mathrm{F}$ & $\mathrm{C}$ & $\mathrm{R}$ & $\mathrm{F}$ & \\
\hline $843 \mathrm{C}-1 \mathrm{H}-1$ & $119-121$ & $A / M-G$ & R & $R$ & & $C$ & & $\mathrm{R}$ & & & $\mathrm{R}$ & $\mathrm{R}$ & $R$ & & $\mathrm{~F}$ & $\mathrm{~F}$ & & & & & A & \\
\hline $843 \mathrm{C}-1 \mathrm{H}-2$ & $19-21$ & $A / M-G$ & & $\mathrm{~F}$ & & C & A & $\mathrm{F}$ & & & $R$ & & $\mathrm{~F}$ & & $R$ & $\mathrm{~F}$ & & $R$ & & & A & \\
\hline $843 \mathrm{C}-1 \mathrm{H}-2$ & 129-131 & C/P-M & & & & & $R$ & $\mathrm{R}$ & & & $R$ & & & & $\mathrm{R}$ & & & & & & $\mathrm{F}$ & \\
\hline $843 \mathrm{C}-1 \mathrm{H}-3$ & $19-21$ & $A / M-G$ & & $\mathrm{~F}$ & & $\mathrm{~F}$ & $C$ & $C$ & $\mathrm{R}$ & & & & $\mathrm{F}$ & & $\mathrm{R}$ & & & $\mathrm{R}$ & & & $\mathrm{F}$ & \\
\hline
\end{tabular}

Note: Letter codes are explained in the "Sample and Data Preparation" section (this chapter).

\section{Site 843}

\section{Hole $843 \mathrm{C}$}

The richest and best preserved Quaternary radiolarian faunas are in Core $843 \mathrm{C}-1 \mathrm{H}$, which recovered $4.2 \mathrm{~m}$ of clays. Surface sediments in Sample $843 \mathrm{C}-1 \mathrm{H}-1,34-36 \mathrm{~cm}$, include more than 70 Quaternary species (Table 1), including several specimens of Collosphaera orthoconus and a single specimen of Collosphaera tuberosa. The surface sediments at Hole $843 \mathrm{C}$ are the same age as the sediments at Hole $842 \mathrm{~B}$, and are assigned to the Collosphaera tuberosa Interval-Zone based on the presence of Collosphaera orthoconus. Axoprunum angelinum, which has its final stratigraphic occurrence in the middle of the Collosphaera tuberosa Interval-Zone ( $0.36 \mathrm{Ma}$ according to recali- brations of Johnson and Knoll, 1975; 0.42 Ma according to Morley and Shackleton, 1978), occurs at 1.20 mbsf in Sample 843C-1H-1, $119-121 \mathrm{~cm}$. The boundary between the Collosphaera tuberosa Interval-Zone (sensu Johnson and Knoll, 1975; Sanfilippo et al., 1985) and the underlying Amphirhopalum ypsilon Interval-Zone is below the occurrence of Axoprunum angelinum at $1.2 \mathrm{mbsf}$. The Brunhes/ Matuyama boundary (which has been correlated elsewhere to the middle of the Amphirhopalum ypsilon Interval-Zone) is at $2.2 \mathrm{mbsf}$. The Jaramillo Event, which has been correlated by other workers to the lower part of the Amphirhopalum ypsilon Interval-Zone, has not been identified in this core. There are no older radiolarian taxa present to suggest that this core is any older than the radiolarian assemblages of the Amphirhopalum ypsilon Interval-Zone (Fig. 2). 
Table 1 (continued).

\begin{tabular}{|c|c|c|c|c|c|c|c|c|c|c|c|c|c|c|c|c|c|c|c|c|c|c|}
\hline $\begin{array}{l}\text { Site, section, } \\
\text { core }\end{array}$ & Interval $(\mathrm{cm})$ & $\begin{array}{l}\text { Abundance/ } \\
\text { Preservation }\end{array}$ & 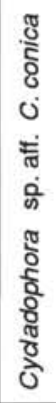 & $\begin{array}{l}\text { के } \\
\text { के } \\
\text { हैँ } \\
\frac{0}{0} \\
\frac{0}{0} \\
\frac{\pi}{0} \\
\text { o }\end{array}$ & $\begin{array}{l}\frac{8}{0} \\
\frac{0}{0} \\
\frac{8}{0} \\
\frac{8}{0} \\
\frac{0}{0} \\
\frac{\pi}{0} \\
0\end{array}$ & $\begin{array}{l}\text { के } \\
\delta \\
8 \\
8 \\
\vdots \\
\vdots \\
0\end{array}$ & 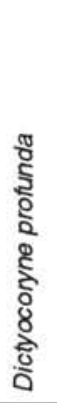 & 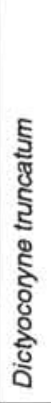 & $\begin{array}{l}\frac{\pi}{0} \\
\frac{0}{5} \\
0 \\
\text { है } \\
\text { है } \\
\frac{0}{0} \\
\frac{0}{0} \\
0 \\
0\end{array}$ & 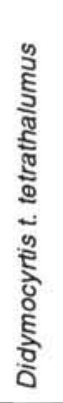 & $\begin{array}{l}\dot{0} \\
\frac{0}{2} \\
\frac{0}{0} \\
\frac{5}{0}\end{array}$ & 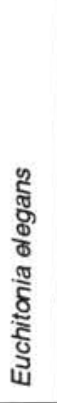 & 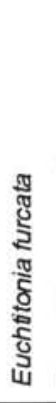 & 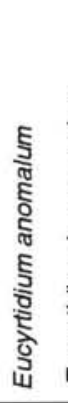 & 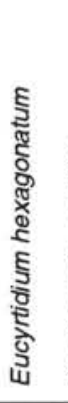 & 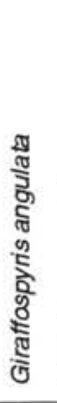 & 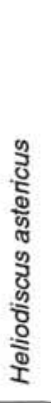 & 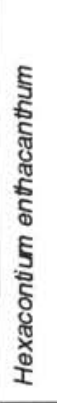 & 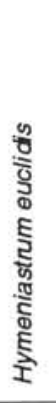 & 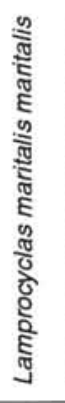 & 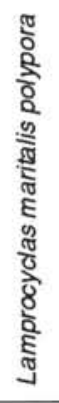 & 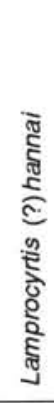 \\
\hline $842 \mathrm{~A}-1 \mathrm{H}-1$ & $28-30$ & A/M-G & & & A & & $A$ & C & $C$ & A & & A & C & & $\mathrm{F}$ & $C$ & C & $A$ & $\mathrm{~F}$ & C & $\mathrm{F}$ & C \\
\hline $842 \mathrm{~A}-1 \mathrm{H}-1$ & $129-131$ & $A / M-G$ & & & A & & $A$ & A & $\mathrm{F}$ & C & & A & C & & $\mathrm{C}$ & & $\mathrm{F}$ & C & $\mathrm{F}$ & $\mathrm{F}$ & & $\mathrm{F}$ \\
\hline $842 \mathrm{~A}-1 \mathrm{H}-2$ & $130-132$ & AVM-G & & & C & & 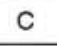 & A & $R$ & c & & C & C & & C & & $\mathrm{C}$ & C & $\mathrm{R}$ & C & & \\
\hline $842 \mathrm{~A}-1 \mathrm{H}-3$ & $7-9$ & AVM-G & & & $\mathrm{F}$ & & A & A & $\mathrm{F}$ & C & & C & C & $R$ & C & $R$ & C & $C$ & & C & C & \\
\hline $842 \mathrm{~A}-1 \mathrm{H}-3$ & $129-131$ & A/M-G & & & c & & C & C & C & C & & C & $\mathrm{F}$ & & C & & $\mathrm{C}$ & C & $R$ & $\mathrm{C}$ & & \\
\hline $842 \mathrm{~A}-1 \mathrm{H}-4$ & $129-131$ & $\mathrm{C} / \mathrm{M}$ & & & $\mathrm{F}$ & & C & C & $R$ & C & & $\mathrm{F}$ & $\mathrm{R}$ & & & $R$ & & $\mathrm{~F}$ & & & & $R$ \\
\hline $842 \mathrm{~A}-1 \mathrm{H}-5$ & $8-10$ & $\mathrm{C} / \mathrm{P}-\mathrm{G}$ & & & & & $\mathrm{C}$ & $\mathrm{C}$ & & $R$ & & A & R & & & & $\mathrm{F}$ & & $\mathrm{F}$ & $\mathrm{F}$ & & \\
\hline $842 \mathrm{~A}-1 \mathrm{H}-5$ & $129-131$ & $C / P-G$ & & & C & $R$ & $\mathrm{C}$ & C & & C & & C & & & c & $R$ & $\mathrm{C}$ & & $R$ & C & & $\mathrm{F}$ \\
\hline $842 \mathrm{~A}-1 \mathrm{H}-6$ & $128-132$ & AVP-M & & & C & & $\mathrm{C}$ & C & $R$ & $C$ & & C & C & & $\mathrm{F}$ & $R$ & & C & $\mathrm{F}$ & $R$ & & \\
\hline $842 \mathrm{~A}-1 \mathrm{H}-7$ & $9-11$ & AVP-M & & & $F$ & $R$ & & C & $R$ & $\mathrm{~F}$ & & $\mathrm{~F}$ & $\mathrm{~F}$ & & & & & C & & & & \\
\hline $842 \mathrm{~B}-1 \mathrm{H}-1$ & $9-11$ & AVM-G & $R$ & & C & & $\mathrm{F}$ & A & $C$ & A & & A & $C$ & & $\mathrm{~F}$ & $\mathrm{~F}$ & $\mathrm{~F}$ & $\mathrm{~F}$ & $\mathrm{~F}$ & $\mathrm{~F}$ & C & \\
\hline $842 \mathrm{~B}-1 \mathrm{H}-1$ & $126-128$ & C/P-G & & & $R$ & $R$ & $A$ & A & & $R$ & & A & C & & $R$ & & & $R$ & $\mathrm{R}$ & & & \\
\hline $842 \mathrm{~B}-1 \mathrm{H}-2$ & $7-9$ & R/P & & & $R$ & & $R$ & R & & & & & & & & & & & & & & \\
\hline $842 \mathrm{~B}-1 \mathrm{H}-2$ & $126-128$ & AVP-G & & & & & $C$ & C & $\mathrm{R}$ & C & & C & C & & $\mathrm{F}$ & $R$ & $C$ & C & & $R$ & & \\
\hline $842 \mathrm{~B}-1 \mathrm{H}-3$ & $9-11$ & AVP-G & & & $R$ & & $F$ & $F$ & $R$ & $R$ & $R$ & C & $R$ & $R$ & $F$ & & $\mathrm{~F}$ & $\mathrm{~F}$ & & & $R$ & \\
\hline $842 \mathrm{~B}-1 \mathrm{H}-3$ & $126-128$ & AVM-G & & & & & $C$ & $F$ & & $R$ & & $C$ & $\mathrm{~F}$ & & $R$ & $R$ & $\mathrm{~F}$ & $\mathrm{~F}$ & $R$ & & & \\
\hline $842 \mathrm{~B}-1 \mathrm{H}-4$ & $9-11$ & Barren & & & & & & & & & & & & & & & & & & & & \\
\hline $842 \mathrm{~B}-1 \mathrm{H}-4$ & $126-128$ & $\mathrm{C} / \mathrm{P}-\mathrm{M}$ & & & & & $\mathrm{F}$ & C & $R$ & $R$ & & $\mathrm{~F}$ & $R$ & & & & & & & & & \\
\hline $842 \mathrm{~B}-1 \mathrm{H}-5$ & $8-10$ & C/M-G & & & $\mathrm{F}$ & & $\mathrm{R}$ & C & $\mathrm{F}$ & $R$ & & & & & & $R$ & $C$ & C & $R$ & & & \\
\hline $843 \mathrm{C}-1 \mathrm{H}-1$ & $34-36$ & $A V G$ & $R$ & $\mathrm{R}$ & A & & $A$ & $C$ & $F$ & $C$ & $\mathrm{R}$ & A & A & $F$ & $C$ & $R$ & $C$ & $C$ & $R$ & $F$ & $R$ & $R$ \\
\hline $843 \mathrm{C}-1 \mathrm{H}-1$ & $119-121$ & A/M-G & & & $C$ & & $C$ & A & $F$ & $\mathrm{~F}$ & & C & $F$ & & $R$ & & $\mathrm{~F}$ & $F$ & R & $R$ & & \\
\hline $843 \mathrm{C}-1 \mathrm{H}-2$ & $19-21$ & A/M-G & & & C & & $C$ & C & $F$ & C & & C & $\mathrm{R}$ & & C & & & & C & $C$ & & $\mathrm{~F}$ \\
\hline $843 \mathrm{C}-1 \mathrm{H}-2$ & $129-131$ & C/P-M & & & & $R$ & $\mathrm{~F}$ & C & & & & C & & & $\mathrm{F}$ & $R$ & & & $R$ & & & \\
\hline $843 \mathrm{C}-1 \mathrm{H}-3$ & $19-21$ & $A / M-G$ & & & $\mathrm{~F}$ & & $F$ & $\mathrm{C}$ & & $\mathrm{F}$ & & C & $F$ & $\mathrm{~F}$ & $\mathrm{C}$ & & $\mathrm{F}$ & $F$ & & $R$ & & \\
\hline
\end{tabular}

\section{Reworked Eocene Radiolarians}

Reworking of radiolarians in the Hawaiian Arch region was noted at DSDP Site 67, approximately $180 \mathrm{~km}$ north of the island of Oahu. Surface sediments at this site contain a mixture of Quaternary and lower to middle Eocene radiolarians (Winterer, Riedel, et al., 1971). At Site 68 on the southwest side of the Hawaiian Arch, coring recovered a $15-\mathrm{cm}$ layer of radiolarian ooze overlying more than 14.5 $\mathrm{m}$ of dark brown phillipsitic clay. This surface ooze was dominated by middle Eocene radiolarians mixed with Quaternary species; rare Eocene radiolarians were recovered from the underlying clay (Tracey, Sutton, et al., 1971).

Initial shipboard observations of samples recorded the presence of the reworked Pliocene species Spongaster pentas in Samples $842 \mathrm{~A}-1 \mathrm{H}-3,7-9 \mathrm{~cm}(3.07-3.09 \mathrm{mbsf})$, and $842 \mathrm{~A}-1 \mathrm{H}-3,129-131 \mathrm{~cm}$
(4.29-4.31 mbsf). Post-cruise examinations of three samples collected from layers of volcanic sand in Core $842 \mathrm{~A}-1 \mathrm{H}$ have yielded an assemblage of poorly to moderately preserved early to middle Eocene radiolarians. These reworked radiolarian faunas occur with relatively well-preserved Quaternary assemblages, and appear to be confined primarily to the volcanic sand layers. Repeated observations of samples collected in close proximity or adjacent to these layers have failed to find Eocene radiolarians, but the presence of bioturbation suggests rare occurrences are possible elsewhere in the core (Garcia, pers. comm., 1992). The most diverse assemblage is from Sample 842A1H-6, 66-67 cm, which includes Calocyclas hispida, Buryella tetradica, Giraffospyris lata, Phormocyrtis striata striata, Phormocyrtis striata exquisita, Podocyrtis (Lampterium) sp. aff. P. (L.) acalles, Podocyrtis (Lampterium) sinuosa, Podocyrtis (Podocyrtis) papalis, Theocotylissa alpha, Stylotrochus quadribrachiatus quadribra- 
Table 1 (continued).

\begin{tabular}{|c|c|c|c|c|c|c|c|c|c|c|c|c|c|c|c|c|c|c|c|c|c|c|}
\hline $\begin{array}{l}\text { Site, section, } \\
\text { core }\end{array}$ & Interval (cm) & $\begin{array}{l}\text { Abundance/ } \\
\text { Preservation }\end{array}$ & 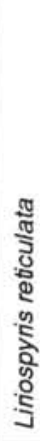 & 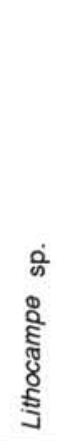 & 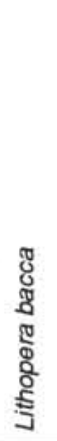 & 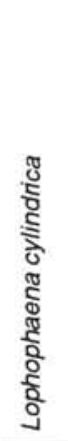 & 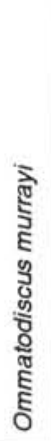 & 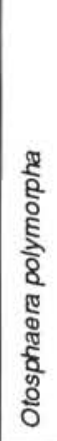 & 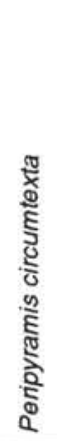 & 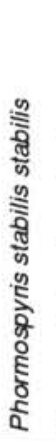 & 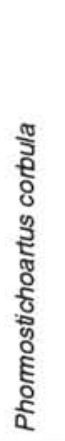 & 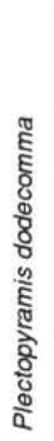 & 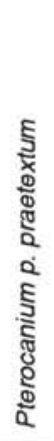 & 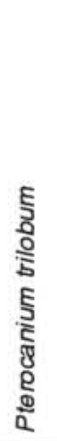 & 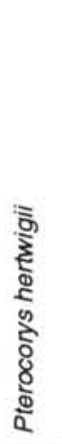 & 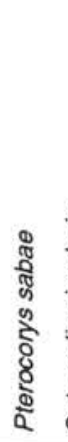 & 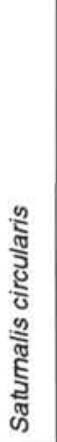 & 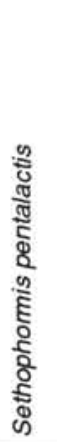 & 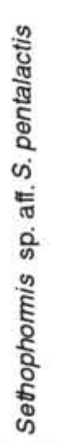 & 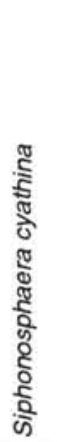 & 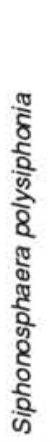 & 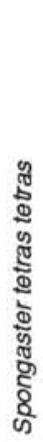 \\
\hline $842 \mathrm{~A}-1 \mathrm{H}-1$ & $28-30$ & A/M-G & $\mathrm{C}$ & $F$ & $A$ & $R$ & & & C & & $R$ & & C & A & $R$ & & C & & & $\mathrm{F}$ & A & A \\
\hline $842 A-1 H-1$ & $129-131$ & A/M-G & $\mathrm{F}$ & $\mathrm{F}$ & C & & $R$ & & & & & & $F$ & $c$ & $\mathrm{~F}$ & & $\mathrm{~F}$ & & $R$ & & $\mathrm{~F}$ & $\mathrm{~F}$ \\
\hline $842 \mathrm{~A}-1 \mathrm{H}-2$ & $130-132$ & AVM-G & $F$ & & & & C & & C & & $\mathrm{F}$ & & $\mathrm{F}$ & C & & & $R$ & & & R & $\mathrm{F}$ & $F$ \\
\hline $842 \mathrm{~A}-1 \mathrm{H}-3$ & $7-9$ & $A / M-G$ & $\mathrm{~F}$ & $\mathrm{~F}$ & $\mathrm{C}$ & & & & 7 & & & $R$ & $R$ & & $R$ & & & & & $\mathrm{~F}$ & $\mathrm{~F}$ & $\mathrm{C}$ \\
\hline $842 \mathrm{~A}-1 \mathrm{H}-3$ & $129-131$ & $A / M-G$ & & $\mathrm{~F}$ & $\mathrm{C}$ & & & & & $R$ & & $R$ & C & C & & & C & & & C & C & C \\
\hline $842 \mathrm{~A}-1 \mathrm{H}-4$ & $129-131$ & $\mathrm{C} / \mathrm{M}$ & $R$ & & $\mathrm{~F}$ & & & & $\mathrm{~F}$ & & & & $R$ & $\mathrm{~F}$ & & & $\mathrm{~F}$ & & & & & $R$ \\
\hline $842 \mathrm{~A}-1 \mathrm{H}-5$ & $8-10$ & C/P-G & & & & & & & $\mathrm{R}$ & & & $R$ & & & & & C & & & & & $\mathrm{F}$ \\
\hline $842 \mathrm{~A}-1 \mathrm{H}-5$ & $129-131$ & C/P-G & $R$ & & C & & & & & & $R$ & $\mathrm{~F}$ & $F$ & $\mathrm{~F}$ & & & $F$ & & & & $R$ & $\mathrm{~F}$ \\
\hline $842 \mathrm{~A}-1 \mathrm{H}-6$ & $128-132$ & AVP-M & & & C & & $\mathrm{F}$ & & & R & & $\mathrm{F}$ & & $\mathrm{F}$ & & & $\mathrm{F}$ & $R$ & $R$ & & $F$ & A \\
\hline $842 \mathrm{~A}-1 \mathrm{H}-7$ & $9-11$ & AVP-M & $R$ & & $\mathrm{~F}$ & & & & $\mathrm{~F}$ & & & $R$ & & $\mathrm{~F}$ & & & C & & $R$ & & & C \\
\hline $842 \mathrm{~B}-1 \mathrm{H}-1$ & $9-11$ & $A / M-G$ & $\bar{R}$ & $\mathrm{C}$ & $A$ & & $R$ & & $\mathrm{C}$ & & & & & $R$ & $F$ & & $R$ & & & & $A$ & $F$ \\
\hline $842 \mathrm{~B}-1 \mathrm{H}-1$ & $126-128$ & C/P-G & R & $R$ & & & $F$ & $R$ & & & & & & $R$ & $\mathrm{R}$ & & $\mathrm{C}$ & & $R$ & & $R$ & A \\
\hline $842 \mathrm{~B}-1 \mathrm{H}-2$ & $7-9$ & R/P & & & & & & & & & & & & & & & & & & & & \\
\hline $842 \mathrm{~B}-1 \mathrm{H}-2$ & $126-128$ & ANP-G & $F$ & & $R$ & & $F$ & & C & $R$ & & & & C & & & $C$ & & $R$ & & $\mathrm{~F}$ & $F$ \\
\hline $842 \mathrm{~B}-1 \mathrm{H}-3$ & $9-11$ & ANP-G & $R$ & $R$ & $C$ & & $R$ & $R$ & $R$ & & $R$ & & $R$ & $\mathrm{~F}$ & & & $R$ & & & & $\mathrm{~F}$ & R \\
\hline $842 \mathrm{~B}-1 \mathrm{H}-3$ & $126-128$ & AVM-G & $R$ & $R$ & 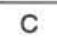 & $R$ & $R$ & & & & R & & C & & $R$ & & $\mathrm{~F}$ & & & & $R$ & \\
\hline $842 \mathrm{~B}-1 \mathrm{H}-4$ & $9-11$ & Barren & & & & & & & & & & & & & & & & & & & & \\
\hline $842 \mathrm{~B}-1 \mathrm{H}-4$ & $126-128$ & C/P-M & & & & & $R$ & & $R$ & & & & $R$ & & & & $\mathrm{~F}$ & & & & & C \\
\hline $842 \mathrm{~B}-1 \mathrm{H}-5$ & $8-10$ & $\mathrm{C} / \mathrm{M}-\mathrm{G}$ & $\mathrm{F}$ & & $C$ & & $F$ & & $F$ & & & $R$ & & $R$ & & & $R$ & & & & $\mathrm{~F}$ & C \\
\hline $843 \mathrm{C}-1 \mathrm{H}-1$ & $34-36$ & AVG & $\bar{F}$ & & C & & & $R$ & $R$ & $R$ & $\mathrm{~F}$ & & $\mathrm{~F}$ & C & C & & $R$ & & & $R$ & $C$ & R \\
\hline $843 \mathrm{C}-1 \mathrm{H}-1$ & $119-121$ & AVM-G & $R$ & $R$ & $\mathrm{~F}$ & & & & $\mathrm{~F}$ & & R & $R$ & $R$ & $\mathrm{~F}$ & & $R$ & $R$ & & & & & $F$ \\
\hline $843 \mathrm{C}-1 \mathrm{H}-2$ & $19-21$ & $\mathrm{~A} / \mathrm{M}-\mathrm{G}$ & $\mathrm{F}$ & & & & & & $\mathrm{F}$ & $R$ & $R$ & $\mathrm{~F}$ & & $\mathrm{~F}$ & & & $F$ & & & $R$ & $\mathrm{~F}$ & R \\
\hline $843 \mathrm{C}-1 \mathrm{H}-2$ & $129-131$ & C/P-M & $\mathrm{F}$ & & & & & & & & & & $R$ & & & & & $R$ & & & $R$ & \\
\hline $843 \mathrm{C}-1 \mathrm{H}-3$ & $19-21$ & $A / M-G$ & $\mathrm{~F}$ & & $\mathrm{~F}$ & & $R$ & & $\mathrm{R}$ & & R & & $\mathrm{F}$ & $\mathrm{F}$ & $R$ & & $\mathrm{~F}$ & $R$ & & $R$ & $\mathrm{~F}$ & \\
\hline
\end{tabular}

chiatus, and Thyrsocyrtis (Pentalacorys) triacantha. All of these species range from early to middle Eocene. Sample 842A-1H-6, $124-126 \mathrm{~cm}$, contains the following Eocene radiolarians: Podocyrtis (Lampterium) acalles, Thyrsocyrtis (Pentalacorys) triacantha, and Giraffospyris lata. Theocotylissa alpha, Theocotylissa ficus, and an unnamed species of Podocyrtis (Podocyrtis) occur in Sample 842A$1 \mathrm{H}-7,6-8 \mathrm{~cm}$.

The source of Eocene radiolarians at Site 842 during the early to middle Quaternary is an interesting question. Eocene radiolarians have not been recovered either in situ from cored intervals in Hole $842 \mathrm{~B}$, or from wash cores at Site 843 . It is unlikely that the Eocene assemblages are reworked upsection from such great depths at the same site. Furthermore, the relative abundance of Eocene radiolarians in the volcanic sand layers suggests simultaneous transport of Quaternary and Eocene radiolarians and sands across the surface of the seafloor during deposition. Riedel and Funnell (1964) attribute the reworking of Eocene radiolarians in the central Pacific region to bottom currents. Schreiber (1969) is the first to propose a turbidite origin for the mixed sediments; Garcia (this volume) also thinks that the volcanic sand layers are turbidites, related to giant submarine slides that have scoured into the Eocene portion of the seafloor. Regardless of the mechanism, evidence for reworking of ichthyoliths in Cores $842 \mathrm{~B}-3 \mathrm{H}$ through $842 \mathrm{~B}-5 \mathrm{H}$ (Firth and Hull, this volume) suggests that such deep-sea processes may have been active in this region throughout the Neogene.

\section{Cretaceous Radiolarian Biostratigraphy}

Chert samples collected from the lowermost cored intervals at Sites 842 and 843 were analyzed for radiolarians. Species identifica- 
Table 1 (continued).

\begin{tabular}{|c|c|c|c|c|c|c|c|c|c|c|c|c|c|c|c|c|c|}
\hline $\begin{array}{l}\text { Site, section, } \\
\text { core }\end{array}$ & Interval (cm) & $\begin{array}{l}\text { Abundance/ } \\
\text { Preservation }\end{array}$ & 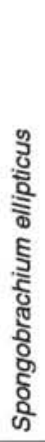 & 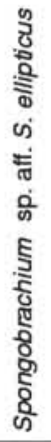 & 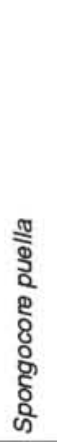 & 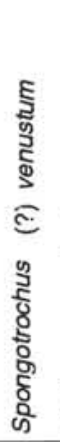 & 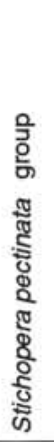 & 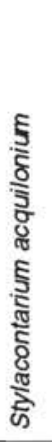 & 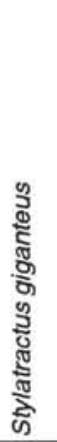 & $\begin{array}{l}\text { के } \\
\text { के } \\
\text { ญू } \\
\text { कू } \\
\text { के }\end{array}$ & 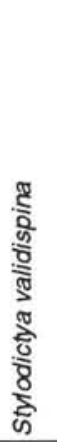 & 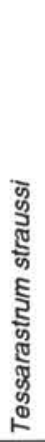 & 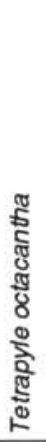 & 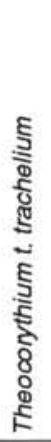 & 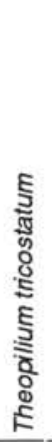 & 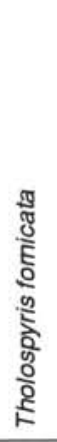 & 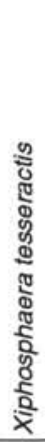 \\
\hline $842 \mathrm{~A}-1 \mathrm{H}-1$ & $28-30$ & A/M-G & & $R$ & A & & $R$ & C & $R$ & & A & C & $\mathrm{F}$ & C & & & \\
\hline $842 \mathrm{~A}-1 \mathrm{H}-1$ & $129-131$ & $A / M-G$ & & & A & $\mathrm{R}$ & & & $\mathrm{R}$ & $R$ & $C$ & & c & A & $\mathrm{R}$ & & \\
\hline $842 \mathrm{~A}-1 \mathrm{H}-2$ & $130-132$ & A/M-G & & & C & & & C & & & $C$ & & $\mathrm{~F}$ & C & & & \\
\hline $842 \mathrm{~A}-1 \mathrm{H}-3$ & $7-9$ & A/M-G & & & C & & & $\mathrm{F}$ & $\mathrm{F}$ & $R$ & $C$ & $R$ & $\mathrm{~F}$ & C & & & \\
\hline $842 \mathrm{~A}-1 \mathrm{H}-3$ & $129-131$ & A/M-G & R & & C & $\mathrm{R}$ & & $F$ & $\mathrm{R}$ & C & $C$ & & $\mathrm{~F}$ & $\mathrm{~F}$ & C & & \\
\hline $842 \mathrm{~A}-1 \mathrm{H}-4$ & $129-131$ & $\mathrm{C} / \mathrm{M}$ & & & c & & $R$ & c & & & $F$ & & & $\mathrm{~F}$ & & & \\
\hline $842 \mathrm{~A}-1 \mathrm{H}-5$ & $8-10$ & C/P-G & & & C & & & $\mathrm{F}$ & $\mathrm{R}$ & & $\mathrm{F}$ & & & & & & \\
\hline $842 \mathrm{~A}-1 \mathrm{H}-5$ & $129-131$ & C/P-G & & & C & & $R$ & $\mathrm{~F}$ & & & $\mathrm{~F}$ & & R & $R$ & & & \\
\hline $842 \mathrm{~A}-1 \mathrm{H}-6$ & $128-132$ & AVP-M & & & C & $R$ & $\mathrm{~F}$ & $\mathrm{~F}$ & & $R$ & & & & $\mathrm{~F}$ & $R$ & & \\
\hline $842 \mathrm{~A}-1 \mathrm{H}-7$ & $9-11$ & AVP-M & R & & C & & & & & & C & & C & & & & \\
\hline $842 \mathrm{~B}-1 \mathrm{H}-1$ & $9-11$ & A/M-G & & & $\mathrm{F}$ & $\mathrm{R}$ & & & & & $\mathrm{F}$ & $\mathrm{F}$ & C & C & & & \\
\hline $842 \mathrm{~B}-1 \mathrm{H}-1$ & $126-128$ & $\mathrm{C} / \mathrm{P}-\mathrm{G}$ & & $R$ & C & $\mathrm{R}$ & & R & $R$ & & $\mathrm{~F}$ & & $R$ & & & & \\
\hline $842 \mathrm{~B}-1 \mathrm{H}-2$ & $7-9$ & R/P & & & & & & & & & $R$ & & & & & & \\
\hline $842 \mathrm{~B}-1 \mathrm{H}-2$ & $126-128$ & AVP-G & $R$ & & C & & & $R$ & & & $C$ & & $R$ & C & $R$ & & R \\
\hline $842 \mathrm{~B}-1 \mathrm{H}-3$ & $9-11$ & AVP-G & R & & $F$ & & & & $R$ & & $\mathrm{~F}$ & & & $R$ & $\mathrm{~F}$ & & \\
\hline $842 \mathrm{~B}-1 \mathrm{H}-3$ & $126-128$ & A/M-G & & & $\mathrm{F}$ & & & R & & & C & & R & $R$ & & & \\
\hline $842 \mathrm{~B}-1 \mathrm{H}-4$ & $9-11$ & Barren & & & & & & & & & & & & & & & \\
\hline $842 \mathrm{~B}-1 \mathrm{H}-4$ & $126-128$ & C/P-M & & & $F$ & & $R$ & $R$ & & & $R$ & & & $R$ & & & \\
\hline $842 \mathrm{~B}-1 \mathrm{H}-5$ & $8-10$ & $\mathrm{C} / \mathrm{M}-\mathrm{G}$ & & & & $R$ & & $\mathrm{~F}$ & & & 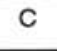 & $R$ & $\mathrm{~F}$ & & & & \\
\hline $843 \mathrm{C}-1 \mathrm{H}-1$ & $34-36$ & AGG & & & $\mathrm{F}$ & $\mathrm{R}$ & $\mathrm{F}$ & R & $R$ & $R$ & $F$ & $\mathrm{~F}$ & $\mathrm{~F}$ & & $\mathrm{~F}$ & $R$ & \\
\hline $843 \mathrm{C}-1 \mathrm{H}-1$ & $119-121$ & A/M-G & & & $\mathrm{F}$ & $\mathrm{F}$ & & $R$ & $R$ & & $\mathrm{~F}$ & & $\mathrm{~F}$ & R & & & \\
\hline $843 \mathrm{C}-1 \mathrm{H}-2$ & $19-21$ & A/M-G & & & & $\mathrm{F}$ & & $R$ & & & $F$ & & $\mathrm{~F}$ & $\mathrm{~F}$ & $R$ & & \\
\hline $843 \mathrm{C}-1 \mathrm{H}-2$ & $129-131$ & C/P-M & & $R$ & & & & & & & $\mathrm{~F}$ & & & & & & \\
\hline $843 \mathrm{C}-1 \mathrm{H}-3$ & $19-21$ & $A / M-G$ & R & & $\mathrm{F}$ & & & R & & & $\mathrm{F}$ & & C & C & & & R \\
\hline
\end{tabular}

tions and age determinations were derived from Pessagno (1976, 1977). Overall preservation of Cretaceous radiolarians in these samples ranged from moderate to poor.

Samples 842B-6X-CC (35.7-39.2 mbsf), 842B-7X-CC, 8-9 cm (40.1 mbsf), and 842B-8X-CC (48.7-58.1 mbsf) are barren of all microfossils, with the exception of abundant sponge spicules in the latter sample. A wash core collected between $842 \mathrm{~B}-8 \mathrm{X}$ and $842 \mathrm{~B}-9 \mathrm{X}$ contains very poorly preserved radiolarians, including specimens of the Paleocene genus Bekoma, and rare Upper Cretaceous forms, ranging in age from Cenomanian to Coniacian. Poorly preserved Upper Cretaceous radiolarians were also extracted from chert associ- ated with nannofossil ooze in Core 842B-10X-CC (163.3-137.8 mbsf). This assemblage includes Alievium murphyi (Coniacian?; Santonian to Maastrichtian) and Dictyomitra densicostata (Coniacian to Campanian). Abundant nannofossils within this same core indicate an early Santonian age (Dziewonski, Wilkens, Firth, et al., 1992).

Less than $1 \mathrm{~m}$ of chert rubble was recovered from Core $842 \mathrm{C}-1 \mathrm{~W}$. Two samples were examined from this interval: $842 \mathrm{C}-1 \mathrm{~W}-1,65-67$ $\mathrm{cm}$ (142.0 mbsf) and Sample 842C-1W-1, 96-97 cm (142.3 mbsf). The latter sample is barren of all microfossils. The former sample contains a moderately well-preserved assemblage of Upper Cretaceous radiolarians, including Archaeodictyomitra sliteri, Pseudodic- 


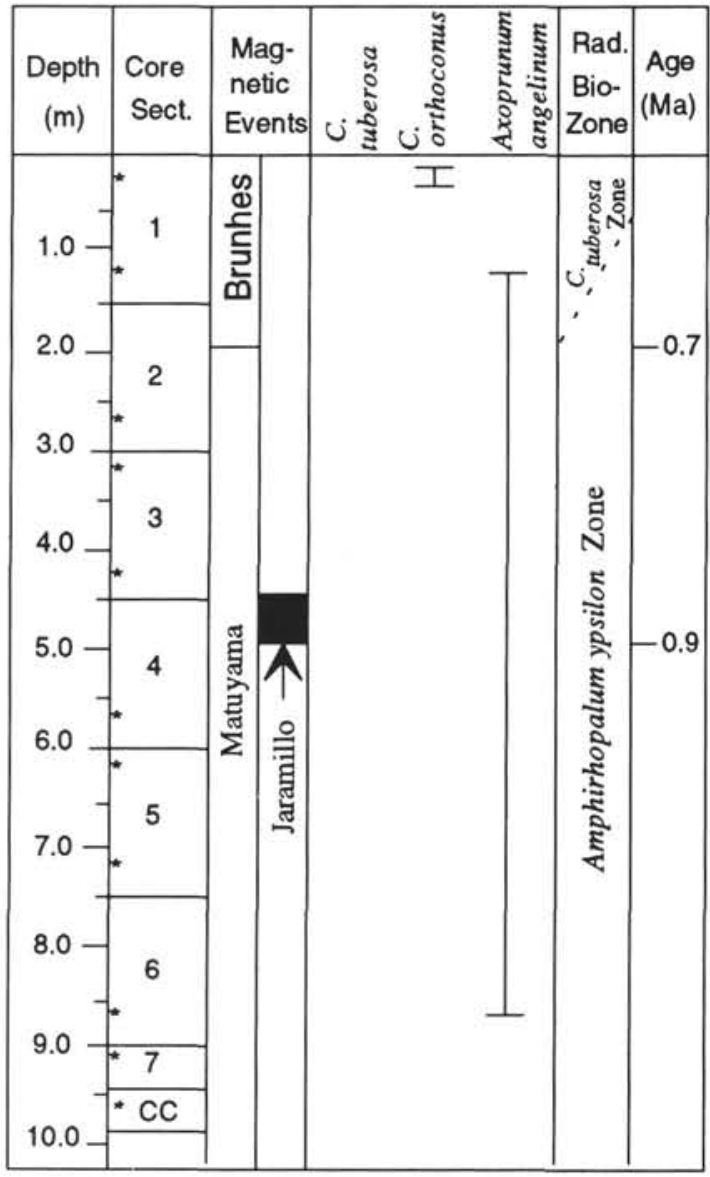

Core $842 \mathrm{~A}-1 \mathrm{H}$

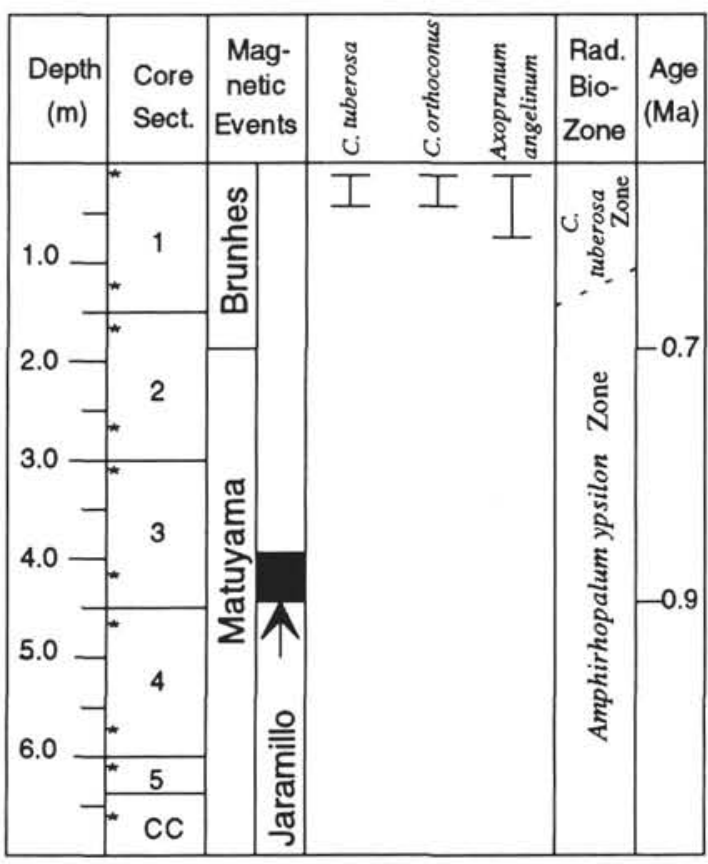

Core $842 \mathrm{~B}-1 \mathrm{H}$

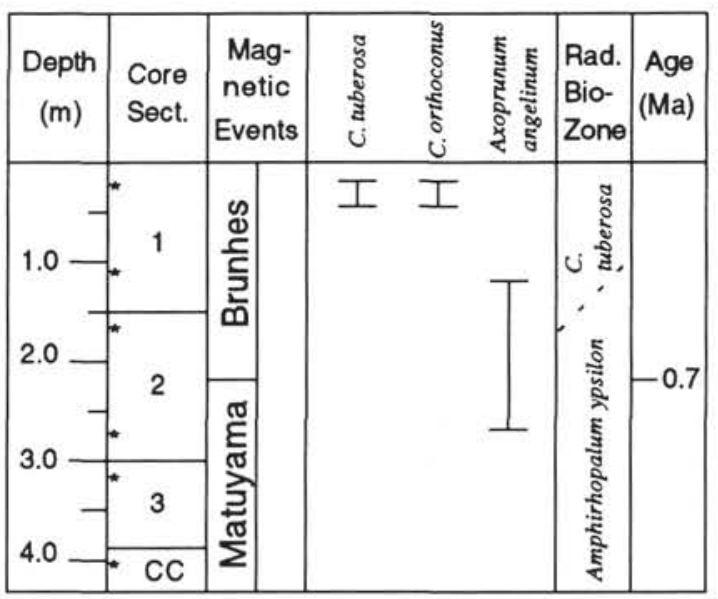

Core $843 \mathrm{C}-1 \mathrm{H}$

Figure 2. Magnetostratigraphy and radiolarian biostratigraphy in Cores $842 \mathrm{~A}-1 \mathrm{H}, 842 \mathrm{~B}-1 \mathrm{H}$, and $843 \mathrm{C}-1 \mathrm{H}$. Radiolarian zonal assignments are based on the occurrence(s) of age-diagnostic taxa in each core and the zonation scheme of Nigrini (1971), as modified by Johnson and Knoll (1975; see also Sanfilippo et al., 1985). Asterisks mark locations of samples.

tyomitra pseudomacrocephala, Novixitus mclaughlini, and several unnamed species. The assemblage is assignable to lower Cenomanian Zone 10, Subzone 10A of Pessagno (1977).

Four cherts were analyzed from Core 843A-3R, which recovered the sediment/basement contact. Unfortunately, all of the cherts were barren, although nannofossil ooze directly overlying the basalt in this core provides an age determination of late Albian to early Cenomanian (Dziewonski, Wilkens, Firth, et al., 1992).

\section{TAXONOMY}

Quaternary taxonomy is presented as a species list, with species names followed by plate number(s), figure number(s) and remarks. Numerous studies of Quaternary radiolarians contain well-documented synonymies, which are not repeated here. Synonymies for most of the following taxa can be found in: Nigrini (1967, 1968, 1970), Riedel and Sanfilippo (1971, 1978), Petrushev- skaya and Kozlova (1972), Dinkelman (1973), Kling (1973), and Nigrini and Moore (1979). The Family Collosphaeridae Müller has been reviewed and revised by Strelkov and Reshetnjak (1971) and by Bjørklund and Goll (1979). Goll $(1968,1969,1976)$ has undertaken extensive studies on the Family Trissocyclidae Haeckel. Tropical forms of the Family Artostrobiidae Riedel are described by Nigrini (1977). More recent taxonomic publications of importance include, among others, Caulet and Nigrini (1988) and Lombari and Lazarus (1988). Taxonomic history is provided under the heading "Remarks" for species whose synonymies cannot be found in any of these publications. Synonymies are provided herein for both Eocene and Cretaceous species documented on Leg 136.

\section{Quaternary Species List}

Acrosphaera flammabunda (Haeckel) (Pl. 1, Fig. 7)

Remarks: The genus Acrosphaera Haeckel (1881) is treated herein as synonymous with Polysolenia Ehrenberg (Campbell, 1954, p. D52). 
Bjørklund and Goll (1979), following Haeckel (1881), rejected use of the genus name Polysolenia based on inadequate definition of the genus and disagreement on its type species. Until the genus Polysolenia is emended and agreement is reached on its type species, this author follows Bjørklund and Goll (1979) in their synonymy of Polysolenia and Acrosphaera.

Acrosphaera lapaccea (Haeckel) (PI. 1, Fig. 11)

Acrosphaera murrayana (Haeckel) (PI. 1, Fig. 15)

Acrosphaera spinosa (Haeckel) (PI. 1, Figs. 8, 9)

Collosphaera macropora Popofsky (Pl. 1, Fig. 6)

Collosphaera orthoconus (PI. 1, Figs. 18, 19)

Collosphaera tuberosa Haeckel (PI. 1, Fig. 16)

Collosphaera spp. (Pl. 1, Figs. 1, 2, 10)

Disolenia sp. (Pl. 1, Fig. 17)

Otosphaera polymorpha Haeckel (PI. 1, Fig. 12)

Remarks: Bjørklund and Goll (1979, p. 1321; = their Trisolenia megalactis megalactis broadened the definition of this species to include several other morphotypes currently assigned to four separate genera. The similarities between these various forms is recognizable, and it may be advisable to bring them together under one genus name rather than one species name. The distinction and stability of the species $O$. polymorpha is maintained herein until complete studies are undertaken to better define the systematics of the Collosphaeridae.

Siphonosphaera cyathina Haeckel (PI. 1, Fig. 3)

Remarks: This species is characterized by short, closely spaced tubules on a large portion of the test surface. It was originally defined by Haeckel (1887, p. 105, pl. 6, fig. 10).

Siphonosphaera polysiphonia Haeckel (PI. 1, Figs. 4, 5)

Axoprunum angelinum (Campbell and Clark) (PI. 1, Fig. 21)

Remarks: Specimens possessing both short and long primary spines were included in A. angelinum by Kling (1973, pl. 1, figs. 13-16). Specimens with long, stout polar spines are extremely rare in these cores.

Axoprunum stauraxonium Haeckel (PI. 1, Fig. 20)

Remarks: Kling (1973, pl. 14, figs. 3, 4) illustrated "lighter-shelled" specimens of Stylacontarium acquilonium (Hays) that are nearly identical to the specimen described herein as A. stauraxonium. The two species possess many characteristics in common, and both thick- and thin-walled individuals have been included under both species names (compare description of A. stauraxonium in Hays, 1965, p. 170-171 with the description of Drupputractus acquilonius in Hays, 1970, p. 214). In this study, the specimen figured on Plate 1, Figure 20, has been assigned to $A$. stauraxonium on the basis of Haeckel's original description and illustration of this species.

Stylacontarium acquilonium (Hays) (PI. I, Fig. 22)

Remarks: This form differs slightly from the original description of Stylacontarium acquilonium (Hays, 1970, p. 214, pl. 1, figs. 4, 5) by possessing bipolar spines that remain weakly triradiate throughout the length of the spine, rather than becoming cylindrical distally.

Stylatractus giganteus Haeckel (Pl. 1, Fig. 14)

Remarks: Original illustration of this species is from Haeckel (1887, p. 329 , pl. 1 fig. 14)

Stylatractus sp. (Pl. 1, Fig. 13)

Remarks: This specimen differs slightly from Stylatractus sp. figured in Nigrini and Moore (1979, p. S55, pl. 7, figs. 1a, b) by possessing less robust spines that are more distinctly triradiate.

Hexacontium enthacanthum Haeckel (Pl. 6. Fig. 7)

Xiphosphaera tesseractis Dreyer (Pl. 2, Fig. 12)

Remarks: The original reference to $X$. tesseractis is from Dreyer (1913, p. 10, pl. 2, figs. 3, 3a). It has also been illustrated by Renz (1976. pl. 2, fig. 2).

Didymocyrtis tetrathalamus tetrathalamus (Haeckel) (Pl. 3, Fig. 5)

Remarks: D. tetrathalamus tetrathalamus (Haeckel) is described under the generic name Ommartartus in Nigrini and Moore (1979, p. S49). The genus was first emended by Riedel (1971) and later by Sanfilippo and Riedel (1980).

Saturnalis circularis Haeckel (Pl. 2, Fig. 5)

Spongocore puella Haeckel (PI. 3, Figs. 8, 9)

Heliodiscus astericus Haeckel (PI. 2. Fig. 4)

Amphirhopalum ypsilon Haeckel (Pl. 2, Figs. 1, 6)

Euchitonia elegans (Ehrenberg) (Pl. 2, Figs. 9, 10)

Euchitonia furcata Ehrenberg (PI. 2, Fig. 11)

Dictyocoryne profunda Ehrenberg (PI. 2, Fig. 16)

Dictyocoryne truncatum (Ehrenberg) (Pl. 2, Fig. 2)

Dictyocoryne sp. aff. D. truncatum (Ehrenberg) (PI. 2, Fig. 13)

Remarks: This form differs from Dictyocoryne truncatum (Ehrenberg) by having three rays which Ling and Anikouchine (1967, p. 1489) described as "chalice-shaped." The arms are also distinguished by a thin rim surrounding the periphery of the test. Nigrini and Moore (1979, p. S89) included this form in their synonymy of $D$. truncatum; it remains separated herein, although the forms are likely related.

Hymeniastrum euclidis Haeckel (Pl. 2, Fig. 14)

Hymeniastrum sp. aff. H. euclidis Haeckel (PI. 2, Fig. 7)

Remarks: Hymeniastrum sp. aff. $H$. euclidis possesses shorter rays and more bulbous tips than the species $H$. euclidis. The central area of this specimen is obscured by dense, spongy meshwork. This morphotype is relatively common throughout the cores.

Tessarastrum straussi Haeckel (Pl. 4, Fig. 16)

Remarks: Tessarastrum straussi Haeckel was originally described by Haeckel (1887, p. 547, pl. 45, fig. 8) and subsequently illustrated by Renz (1976; p. 113, pl. 3, fig. 7).

Spongaster tetras tetras Ehrenberg (Pl. 3, Fig. 1)

Spongotrochus(?) venustum (Bailey) (PI. 3, Fig. 7)

Spongobrachium ellipticum Haeckel (PI. 2, Fig. 3)

Remarks: The original reference to S. ellipticum is Haeckel (1862, p. 461).

Spongobrachium sp. aff. S. ellipticum Haeckel (PI. 2, Fig. 15)

Remarks: This morphotype is also figured by Renz (1976, p. 95, pl. 3 , fig. 5).

Ommatodiscus murrayi Dreyer (PI. 3, Fig. 12)

Remarks: Ommatodiscus murrayi was named and illustrated by Dreyer (1889, pl. 9, fig. 56). It has more recently been figured by Cheng and Yeh (1989, p. 185, pl. 1, figs. 15, 18; pl. 9, figs. 14, 15).

Circodiscus microporus (Stöhr) (Pl. 3, Fig. 10)

Remarks: Nigrini and Lombari (1984, p. S73-S74) contains a discussion and synonymy of this species.

Stylodictya validispina Jörgenson (Pl. 3, Fig. 11)

Remarks: An early synonymy of this species can be found in Nigrini and Moore (1979). More recently, the classification of "Stylodictya-type" radiolarians was investigated by Boltovskoy and Vrba (1988).

Tetrapyle octacantha Müller (Pl. 3, Fig. 2)

Giraffospyris angulata (Haeckel) (PI. 6, Fig. 8)

Liriospyris reticulata (Ehrenberg) (Pl. 3, Fig. 3; Pl. 4, Fig. 1).

Phormospyris stabilis stabilis (Goll) (Pl. 4, Fig. 7)

Tholospyris fornicata Popofsky (PI. 6, Fig. 17)

Remarks: Tholospyris fornicata was described originally by Popofsky (1913, p. 309, pl. 30, fig. 2), and has been illustrated by Renz (1976, p. 177, pl. 8, fig. 15).

Tholospyris spp. (PI. 3, Fig. 6; Pl. 6, Figs. 14, 18, 19)

Carpocanistrum spp. (PI. 4, Figs. 4, 8, 9)

Remarks: Numerous forms of this genus, mostly unnamed, are present in Holes 842A, 842B and 843C. Some of these morphotypes have been illustrated by Riedel and Sanfilippo (1971), Nigrini and Moore (1979), and others. Their stratigraphic value has not been determined.

Sethophormis pentalactis Haeckel (PI. 6, Fig. 13)

Remarks: This species was described by Haeckel (1887, p. 1244, pl. 56 , fig. 5). It has been figured more recently by Renz (1976, p. 165, pl. 7 . fig. 7).

Sethophormis sp. aff. S. pentalactis Haeckel (PI. 6, Fig. 11)

Remarks: Note the larger and less regularly arranged polygonal pore frames on the thorax of this morphotype relative to Sethophormis pentalactis Haeckel.

Theopilium tricostatum Haeckel (PI. 4, Fig. 5; Plate 6, Fig. 12)

Remarks: Haeckel (1887) illustrated two morphotypes that are very similar to those figured herein: Theopilium tricostatum (Haeckel, 1887, p. 1322, pl. 70, fig. 6) and Cecryphalium sestrodiscus Haeckel (1887; p. 1399 , pl. 58, fig. 1). The most striking difference between these two species is the presence of three ribs in the wall of the flared thorax in Theopilium tricostatum. External expression of the ribs is absent in Cecryphalium sestrodiscus. Haeckel (1887, p. 1322) also mentions the presence of a thin horn on the cephalis of Theopilium tricostatum, which is, by description, absent on Cecryphalium sestrodiscus. Haeckel's illustration of $C$. sestrodiscus does, however, appear to have a horn (1887, pl. 58, fig. 1).

Cheng and Yeh (1989) figured both morphotypes from the west central Pacific Ocean, and assigned their specimens to Cecryphalium sestrodiscus. Haeckel. Three of their specimens do show the rib structure typical of Theopilium tricostatum Haeckel (see their pl. 11, figs. 7, 14-16). All of the specimens figured in this study possess ribs, and are assigned to $T$. tricostatum. It should be noted that the degree of rib development varies in these specimens, from "weakly developed" to "pronounced," suggesting that there is a very close relationship between this species and Cecry- 
phalium sestrodiscus. Theopilium tricostatum has also been figured by Takahashi and Honjo (1981, pl. 8, fig. 12) from the western Atlantic. Peripyramis circumtexta Haeckel (Pl. 3, Fig. 4)

Plectopyramis dodecomma Haeckel (Pl. 3. Fig. 13)

Dictyophimus crisiae Ehrenberg (PI. 6. Figs. 9. 15)

Pterocanium praetextum praetextum (Ehrenberg) (PI. 4, Fig. 11)

Pterocanium trilobum (Haeckel) (Pl. 6, Fig. 10)

Lophophaena cylindrica (Cleve); emend. Petrushevskaya, 1971 (PI. 4, Fig. 2)

Cycladophora sp. aff. C. conica Lombari and Lazarus (Pl. 5, Figs. 1, 6)

Remarks: These specimens differ from Cycladophora conica Lombari and Lazarus (1988, p. 105-106, pl. 3, figs. 1-16) by possessing slightly shorter horns than described for the species, and very large tetragonal pore frames on the final row of the lower thorax.

Cycladophora sp. cf. C. cabrilloensis (Campbell and Clark) (PI. 4, Fig. 13)

Remarks: Cycladophora cabrilloensis was described by Campbell and Clark (1944, p. 48, pl. 7, figs. 1-3; = Clathrocyclas cabrilloensis) and later figured by Kling (1973, p. 635, pl. 9, figs. 23-25), with subsequent subdivision into subspecies by Lombari and Lazarus (1988). This form has smaller and more numerous pore frames and a less robust apical horn than other previously illustrated specimens.

Cycladophora(?) sp. (PI. 4, Fig. 15)

Remarks: This specimen is questionably assigned to the genus Cycladophora because it does not accurately fit the emended description of Lombari and Lazarus (1988), or that of any other theoperid genus at this time.

Cycladophora spp. (Pl. 5, Figs. 2, 3)

Eucyrtidium sp. aff. E. acuminatum (Ehrenberg) (PI. 6, Figs. 4, 5)

Remarks: The specimen illustrated by Figure 4 possesses a longer and more conical thorax than is typical of the species $E$. acuminatum. The specimen in Figure 5 is differentiated by its more fusiform shape.

Eucyrtidium anomalum (PI. 6, Fig. 6)

Remarks: Eucyrtidium anomalum was described by Haeckel ( 1862 , p. 323, pl. 7, figs. 11-13), and later illustrated by Renz (1976, p. 131, pl. 5 , fig. 8).

Eucyrtidium hexagonatum Haeckel (PI. 6, Fig. 3)

Lithocampe sp. (Pl. 4, Fig. 12)

Cornutella profunda Ehrenberg (PI. 4, Figs. 20, 21)

Stichopera pectinata group (PI. 4, Fig. 19; Plate 6. Fig. 16)

Remarks: See Kling (1973, p. 638) for species included within this group.

Lithopera bacca Ehrenberg (PI. 6, Fig. 2)

Archipilium sp. aff. A. macropus (Haeckel) (PI. 4, Fig. 10)

Clathrocanium sp. (Pl. 4, Fig. 6)

Remarks: This morphotype has also been figured from the Pacific Ocean by Renz (1976, p. 163, pl. 7, fig. 5).

Dictyocodon sp. (PI. 2, Fig. 8)

Anthocyrtidium ophirense (Ehrenberg) (Pl. 5, Fig. 11)

Anthocyrtidium sp. aff. A. zanguebaricum (Ehrenberg) (PI. 5, Fig. 7)

Remarks: The form figured herein possesses a shorter, broader thorax than A. zanguebaricum (Ehrenberg).

Lamprocyclas maritalis maritalis Haeckel (PI. 5, Fig. 14)

Lamprocyclas maritalis polypora Nigrini (PI. 5, Fig. 12)

Lamprocyclas maritalis sp. aff. L.m. polypora Nigrini (PI. 5, Fig. 13)

Lamprocyrtis(?) hannai (Campbell and Clark) (Pl. 5, Fig. 8)

Pterocorys hertwigii (Haeckel) (Pl. 5, Fig. 5)

Pterocorys sabae (Ehrenberg) (Pl. 5, Fig. 10)

Theocorythium trachelium sp. aff. T.t. dianae (Haeckel) (PI. 5, Fig. 4, 9)

Remarks: The abdominal chambers of both specimens narrow distally, unlike the type species of Theocorythium trachelium dianae. The specimen illustrated in Figure 9 also has a more cupola-shaped thorax.

Theocorythium trachelium trachelium (Ehrenberg) (Pl. 6, Fig. 1)

Botryostrobus auritus/australis (Ehrenberg) group (Pl. 4, Fig. 18)

Remarks: Nigrini (1977) contains a discussion on the phylogenetic history of this group of radiolarians.

Botryostrobus sp. aff. B. aquilonaris (Bailey) (Pl. 4, Fig. 14)

Remarks: Test not as robust as $B$. aquilonaris, becoming widest on the third, rather than the fourth, post-cephalic segment.

Botryostrobus sp. (Pl. 4, Fig. 3)

Phormostichoartus corbula (Harting) (Pl. 4, Fig. 17)

\section{Eocene Taxonomy}

Family SPONGURIDAE Haeckel, 1862; emend. Petrushevskaya, 1975 Genus STYLOTROCHUS Haeckel, 1862
Stylochtrochus quadribrachiatus quadribrachiatus Sanfilippo and Riedel (PI. 7, Fig. 11; PI. 8, Fig. 9)

Stylotrochus quadribrachiatus quadribrachiatus Sanfilippo and Riedel, 1973, pl. 14, figs. 1, 2; pl. 31, fig. 1: Moore, 1973, p. 812.

Family TRISSOCYCLIDAE Haeckel, 1881; emend. Goll, 1968

Genus GIRAFFOSPYRIS Haeckel, 1881; emend. Goll, 1969

$$
\text { Giraffospyris lata Goll }
$$

(Pl. 7, Fig. 4; Pl. 8, Fig. 4)

Giraffospyris lata Goll, 1969, p. 334, pl. 58, figs. 22, 24-26; Sanfilippo and Riedel, 1973, p. 529, pl. 18, figs. 3-7, pl. 33, fig. 4; De Wever, P., 1981, pl. 2, fig. 5 .

Family THEOPERIDAE Haeckel, 1881; emend. Riedel, 1967 Genus BURYELLA Foreman, 1973

Buryella tetradica Foreman (Pl. 7, Fig. 2)

Buryella tetradica Foreman, 1973, p. 433, pl. 8, figs. 4, 5, pl. 9, figs. 13, 14; Johnson, 1977, p. 784, pl. 1, fig. 11; Riedel and Sanfilippo, 1978, p. 65, pl. 3, fig. 5; Suyari and Yamasaki, 1988, pl. 1, figs 10. 12; Blome, 1992, p. 644, pl. 1, figs. 7, 8 .

\section{Genus CALOCYCLAS Ehrenberg, 1847b \\ Calocyclas hispida (Ehrenberg) (Pl. 7, Fig. 1)}

Anthocyrtis hispida Ehrenberg, 1873, p. 216; 1875, pl. 8, figs. 2.

Cycladophora hispida (Ehrenberg) Riedel and Sanfilippo, 1970, p. 529, pl. 10, fig. 9; 1971, pl. 3B, figs. 10, 11; Moore, 1971, p. 741, pl. 4, figs. 6, 7; Chen, 1975, p. 459, pl. 3, fig. 10; Suyari and Yamasaki, 1988, pl. 3, fig. 4.

Calocyclas hispida (Ehrenberg) Foreman, 1973, p. 434, pl. 1, figs. 12-15; pl. 9, fig. 18; Moore, 1971, pl. 4, figs. 6, 7; Johnson, 1974, p. 547, pl. 4, fig. 1; Riedel and Sanfilippo, 1978, p. 65, pl. 3, fig. 6.

\section{Genus LYCHNOCANOMA Haeckel, 1887}

\section{Lychnocanoma sp.} (Pl. 8, Fig. 6)

Remarks: The test of this specimen is somewhat similar to Lychnocanoma elongata Vinassa de Regny (1900) illustrated by Sanfilippo et al. (1985, p. 677, Fig. 19, 1b), except that the form figured herein lacks the massive, bladed feet characteristic of that species. Although the feet are broken in this sample, the proximal portions appear to be less massive, weakly bladed to cylindrical.

\section{Genus PHORMOCYRTIS Haeckel, 1887}

Phormocyrtis striata striata Brandt (Pl. 7, Fig. 5)

Phormocyrtis striata Brandt, in Wetzel, 1935, p. 55, pl. 9, fig. 12; Riedel and Sanfilippo, 1970, p. 532, pl. 10, fig. 7; Benson, 1972, p. 1093, pl. 2 , fig. 5. Phormocyrtis striata striata Brandt, Riedel and Sanfilippo, 1971, pl. 8, fig. 4; 1978, p. 71, pl. 7, fig. 11; Foreman, 1973, p. 438, pl. 7, figs. 5, 6, 9; Johnson, 1974 , p. 548, pl. 2, figs. 6, 7; Chen, 1975, p. 456, pl. 3, fig. 8; not Suyari and Yamasaki, 1988, pl. 3, fig. 5; Blome, 1992, p. 645, pl. 2, fig. 6.

\section{Phormocyrtis striata exquisita (Kozlova)}

(Pl. 8, Fig. 12)

Podocyrtis exquisita Kozlova, in Kozlova and Gorbovetz, 1966, p. 106, pl, 17 , fig. 2.

Phormocyrtis striata Brandt, Riedel and Sanfilippo, 1971, pl. 8, fig. 4.

Phormocyrtis striata exquisita (Kozlova) Foreman, 1973, p. 438, pl. 7, figs. 1-4, 7, 8; pl. 12, fig. 5; Johnson, 1977, p. 784, pl. 2, fig. 9; Riedel and Sanfilippo, 1978, p. 71, pl. 7, fig. 10; Nishimura, 1986, pl. 2, fig. 15; Blome, 1992 , p. 645 , pl. 3 , figs. $6,7$. 
Genus RHOPALOCANIUM Ehrenberg, 1847a

Rhopalocanium sp.

(PI. 8, Fig. 5)

Remarks: The specimen figured herein is poorly preserved, but appears to possess features characteristic of this genus. It differs from Rhopalocanium ornatum Ehrenberg, type species of this genus (see Ehrenberg, 1847b, fig. 3), by having a shorter, less massive horn and by having three feet which diverge from the abdomen at or near the base of that chamber.

\section{Genus THEOCOTYLISSA (Foreman), 1973}

Theocotylissa alpha Foreman

(PI. 7, Fig. 10; PI. 8, Figs. 8, 11)

Theocotyle (Theocotylissa) alpha Foreman, 1973, p. 441, pl. 4, figs. 13-15; pl. 12, fig. 16; 1975, p. 621.

Theocotylissa alpha Foreman, Sanfilippo and Riedel, 1982, p. 179, pl. 2, figs. $16-17$.

\section{Theocotylissa ficus Ehrenberg}

(Pl. 8, Fig. 7)

Eucyrtidium ficus Ehrenberg, 1873, p. 288; 1875, p. 70, pl. 11, fig. 19.

Anthocyrtis(?) ficus (Ehrenberg), Bütschli, 1882, pl. 31, fig. 7.

Theoconus ficus (Ehrenberg) Haeckel, 1887, p. 1403.

Theocotyle(?) ficus (Ehrenberg) Riedel and Sanfilippo, 1970, p. 525, pl. 7, figs. 3-5; 1971, p. 1596; Moore, 1971, p. 740, pl. 1, fig. 12.

Theocotyle (Theocotylissa) ficus (Ehrenberg) Foreman, 1973, p. 441, pl. 4. figs. 16-20; 1975, p. 621; Johnson, 1974, p. 549, pl. 4, figs. 10, 11; 1976, p. 436 ; Nigrini, 1974 , p. 1068, pl. 1I, figs. 5-8.

Theocotylissa ficus (Ehrenberg) Sanfilippo and Riedel, 1982, p. 180-181, pl. 2, figs. 19-20.

Genus THYRSOCYRTIS Ehrenberg, 1847b Subgenus THYRSOCYRTIS (PENTALACORYS) Ehrenberg

Thyrsocyrtis (Pentalacorys) triacantha (Ehrenberg) (Pl. 7, Fig. 12; Pl. 8, Fig. 2)

Podocyrtis triacantha Ehrenberg, 1873, p. $254 ; 1875$, p. 82, pl. 13, fig. 4.

Thyrsocyrtis triacantha (Ehrenberg) Riedel and Sanfilippo, 1970, p. 526, pl. 8, figs. 2, 3; 1971, pl. 3C, fig. 7; 1978, p. 82, pl. 10, figs. 10, 11; Moore, 1971, p. 740, pl. 4, fig. 2; Johnson and Parker, 1972, pl. 1, fig. 1; Dinkelman, 1973, p. 787, pl. 2, figs. 7, 8; Foreman, 1973, p. 442, pl. 12, figs. 9-II; Johnson, 1974, p. 549, pl. 5, fig. 16; Holdsworth, 1975, p. 531, pl. 1, fig. 18; Ling, 1975, p. 730, pl. 11, fig. 20.

Thyrsocyrtis (Pentalacorys) triacantha (Ehrenberg) Sanfilippo and Riedel, 1982, p. 176-177, pl. 1, figs. 8-10; pl. 3, figs. 3-4; Suyari and Yamasaki, 1988, pl. 3, fig. 7 .

Family PTEROCORYTHIDAE Haeckel, 1881; emend. Riedel, 1967. emend. Moore, 1972

Genus CRYPTOCARPIUM Sanfilippo and Riedel, 1992

Cryptocarpium sp.

(Pl. 7, Fig. 3)

Remarks: The specimen figured herein is most similar to "Cryptocarpium sp." illustrated by Sanfilippo and Riedel (1992, pl. 3, figs. 7, 8), although the cephalic structure on this specimen is not clearly visible, and the horn, if present, has been broken. According to Sanfilippo and Riedel, this form existed just prior to the evolutionary split off of Podocyrtis (Podocyrtis) papalis Ehrenberg from the Cryptocarpium lineage.

\section{Genus PODOCYRTIS Ehrenberg, 1847a Subgenus PODOCYRTIS (PODOCYRTIS) Ehrenberg}

Podocyrtis (Podocyrtis) papalis Ehrenberg (PI. 7, Figs. 7, 8; Pl. 8, Fig. 10)

Podocyrtis papalis Ehrenberg, 1847b, fig. 2; 1854, pl. 36, fig. 23; 1873, p. 251; Riedel and Hays, 1969, pl. 1, fig. C; ?Petrushevskaya and Kozlova, 1972 , p. 543, pl. 35, fig. 1.

Podocyrtis fasciata Clark and Campbell, 1942, p. 80, pl. 7, figs. 29, 33,

Podocyrtis (Podocyrtis) papalis Riedel and Sanfilippo, 1970, p. 533, pl. 11, fig. 1; Moore, 1971, p. 743, pl. 2, fig. 4; Sanfilippo and Riedel, 1973, p.
531, pl. 20, figs. 11-14; pl. 36, figs. 2, 3; Johnson, 1974, p. 551, pl. 4, fig. 12; Ling, 1975, p. 731, pl. 13, fig. 5.

$$
\text { Podocyrtis (Podocyrtis) sp. aff. P. (P.) papalis Ehrenberg }
$$$$
\text { (PI. 8, Fig. 3) }
$$

Remarks: This form has a more slender test than $P$. $(P$. papalis.

$$
\text { Subgenus PODOCYRTIS (LAMPTERIUM) Haeckel, } 1881
$$

$$
\text { Podocyrtis (Lampterium) sinuosa Ehrenberg }
$$
(Pl. 7, Fig. 9)

[?]Podocyrtis sinuosa Ehrenberg, 1873, p. 253; 1875, pl. 15, fig. 5 . Podocyrtis sinuosa Ehrenberg, Riedel and Hays, 1969, pl. 1, fig. E; 1978, p. 72, pl. 8, fig. 9 .

Podocyrtis (Lampterium) sinuosa Ehrenberg(?), Riedel and Sanfilippo, 1970, p. 534, pl. 11, figs. 3, 4; Moore, 1971, p. 743, pl. 3, fig. 3; Sanfilippo and Riedel, 1973, p. 532, pl. 21, figs. 4, 5.

Podocyrtis (Lampterium) sinuosa Ehrenberg, Johnson, 1974, p. 551, pl. 4, fig. 18; Sanfilippo et al., 1985, p. 698-699, fig. 30.9; Sanfilippo and Riedel, 1992, pl. 3, fig. 6.

\section{Podocvrtis (Lampterium) acalles Sanfilippo and Riedel}

\section{(PI. 8, Fig. 1)}

Podocyrtis (Lampterium) acalles Sanfilippo and Riedel, 1992, p. 12-14, pl. 3 , figs. $2-5$.

Podocyrtis (Lampterium) sp. aff. P. (L.) acalles Sanfilippo and Riedel (PI. 7, Fig. 6)

Remarks: The morphotype figured herein on Plate 7, Figure 6, most closely resembles the "late" morphotype figured by Sanfilippo and Riedel (1992, pl. 3, fig. 5) from the Theocotyle cryptocephala Zone. Its cephalis, however, is smaller than is typical of Podocyrtis (Lampterium) acalles and its thorax is slightly more conical than campanulate in outline, suggesting close affinities with the Podocyrtis (Podocyrtoges) Sanfilippo and Riedel lineage as well. Sanfilippo and Riedel (1992) suggest that these subgenera, both Podocyrtis (Lampterium) acalles and the species Podocyrtis (Podocyrtoges) aphorma separated from the Podocyrtis (Podocyrtis) stock at approximately the same time. If true, differentiation between these two species during the transitional period should be difficult.

\section{Cretaceous Taxonomy}

Family ARCHAEODICTYOMITRIDAE Pessagno, 1976; emend. Pessagno, 1977

Genus ARCHAEODICTYOMITRA Pessagno, 1976; emend. Pessagno, 1977

$$
\text { Archaeodictyomitra sliteri Pessagno }
$$

(PI. 9, Fig. 6)

Dictyomitra costata (Squinabol) Petrushevskaya and Kozlova, 1972, p. 550, pl. 2 , fig. 3 .

Dictyomitra sp. A Foreman, 1975, p. 615, pl. 2G, fig. 18; not pl. 1G, fig. 7; not pl. $2 \mathrm{G}$, figs. 19,20

Archaeodictyomitra sliteri Pessagno, 1977, p. 43-44, pl. 6, figs. 3, 4, 22, 23 , 27; Suyari and Hashimoto, 1985, p. 108, pl. 5, figs. 4, 5; Suyari, 1986, pl. 2, fig. 7, pl. 4, fig. 13, pl. 13, fig. 2; Tumanda, 1989, p. 36, pl. 7, fig. 2.

$$
\text { Archaeodictyomitra sp. aff. A. sliteri Pessagno }
$$

$$
\text { (PI. 9, Fig. 5) }
$$

Remarks: This form is longer and more slender than the type species of A. sliteri Pessagno.

$$
\text { Archaeodictyomitra sp. }
$$

(Pl. 9, Fig. 1)

Remarks: Archaeodictyomitra sp. herein is characterized by a conical test with a relatively large number of thin longitudinal costae. Genus DICTYOMITRA Zittel, 1876; emend. Pessagno, 1976

$$
\text { Dictyomitra sp. cf. D. napaensis Pessagno }
$$

$$
\text { (PI. 9, Figs. 8, 9) }
$$


Remarks: The specimens figured herein have fewer postabdominal chambers and a less pronounced step-like outline than Dictyomitra napaensis Pessagno (1976, p. 53, pl. 4, fig. 16: pl. 5, figs. 1, 9).

\section{Family PSEUDODICTYOMITRIDAE Pessagno, 1977 Genus PSEUDODICTYOMITRA Pessagno, 1977}

Pseudodictyomitra pseudomacrocephala (Squinabol)

(Pl. 9, Figs. 2, 10)

Dictyomitra pseudomacrocephala Squinabol, 1903, p. 139-140, pl. 10, fig. 2; Cita, 1964, p. 142-143, pl. 12, figs. 8-9; Petrushevskaya and Kozlova, 1972, p. 550, pl. 2, fig. 5; Pessagno and Michael, 1974, p. 979; Foreman, 1975, p. 614, pl. 7, fig. 10; Dumitrica, 1975, p. 87-89, text-fig. 2; no. 19; Nakaseko et al., 1979, p. 22, pl. 6, figs. 12-15; Kozlova in Basov et al., 1979, fig. 4.

Dicryomitra malleola Aliev, Pessagno, 1969, p. 610, pl. 5, fig. 8.

Dictyomitra macrocephala Squinabol, Moore, 1973, p. 829, pl. 9, figs. 8, 9; Riedel and Sanfilippo, 1974, pl. 4, figs. 10-11, pl. 14, fig. 11.

Dictyomitra dnistrensis Lozynyak, 1975, p. 52, pl. 2, figs. 3-7.

Dictyomitra(?) pseudomacrocephala Squinabol, Pessagno, 1976, p. 53, pl. 3 , figs. 2-3; Nakaseko et al., 1979, p. 22, pl. 6, figs. 12-15.

Pseudodictyomitra pseudomacrocephala (Squinabol) Pessagno, 1977, p. 51, pl. 8, figs. 10, 11; Schmidt-Effing, 1980, p. 247, fig. 8; Okamura, 1980, pl. 21, fig. 6; De Wever and Thiebault, 1981, p. 592, pl. 1, fig. 5; Nakaseko and Nishimura, 1981, p. 159, pl. 9, figs. 1-4, pl. 16, figs. 5 (not 6), 7, 8; Schaaf, 1981, p. 437, pl. 24, figs. 1a, b; 1984, p. 130; Matsuyama et al., 1982, pl. 2, fig. 3; Mitzutani et al., 1982, p. 70, pl. 4, figs. 1-11; Taketani, 1982, p. 61, pl. 5, figs. 4a, b, pl. 12, figs. 7, 8; Yamauchi, 1982, pl. 1, fig. 13; Nakazawa et al., 1983, pl. 1, fig. 9; Yao, 1984, pl. 5, figs. 11, 12, 16; Sanfilippo et al., 1985, p. 608-609, figs. 10.1a-e; Suyari, 1986, pl. 3, figs. 7,11 , pl. 4 , fig. 1 , pl. 6 , figs. $9-11$, pl. 11 , fig. 3 , pl. 16, fig. 11 .

\section{Pseudodictyomitra sp. aff. P. pentacolaensis Pessagno}

(PI. 9, Fig. 11)

Remarks: This form differs from Pseudodictyomitra pentacolaensis Pessagno (1977, p. 50, pl. 8, figs. 3, 17, 23; pl. 12, fig. 10) by having more widely spaced costae and a slightly broader test.

\section{Family SATURNALIDAE Deflandre, 1953 \\ Genus ACANTHOCIRCUS Squinabol, 1903}

\section{Acanthocircus sp.}

(Pl. 9, Fig, 4)

Family XITIDAE Pessagno, 1977

Genus NOVIXITUS Pessagno, 1977

Novixitus mclaughlini Pessagno (Pl. 9, Fig. 12)

Novixitus mclaughlini Pessagno, 1977, p. 54, pl. 9, fig. 17; Suyari, 1986, pl. 2, fig. 10, not pl. 8, figs. 10, 11; not Kito, 1987, pl. 3, fig. 7 .

\section{Novixitus sp. \\ (PI. 9. Fig. 7)}

Remarks: This test possesses less massive tubercules than other forms of Novixitus Pessagno.

\section{Genus XITUS Pessagno, 1977}

\section{Xitus(?) sp.}

(Pl. 9, Fig. 3)

Remarks: This fragment possesses the test meshwork typical of the genus Xitus, but the absence of the apical portion of the test (and therefore the presence or absence of an apical horn, a key feature in the identification of this genus) makes its assignment to this genus questionable.

\section{ACKNOWLEDGMENTS}

My sincere thanks to C.A. Nigrini and C.D. Blome for their comments and suggestions, which improved an earlier version of this manuscript. J. Firth, M. Garcia, and E.A. Pessagno, Jr., have also contributed through lengthy comments and discussions. I also would like to thank Audrey Meyer (former Manager of Science Operations) and the Ocean Drilling Program for the opportunity to go to sea on the JOIDES Resolution, and The University of Texas at Dallas for their continued support and use of research facilities. This research has been funded by the U.S. Science Support Program, through the Texas A\&M Research Foundation (PO \# 20589).

\section{REFERENCES}

Basov, V.A., Lopatin, B.G., Gramberg, I.S., Danjushevskaya, A.I., Kaban'kov, V.Ya., Lazurkin, V.M., and Patrunov, D.K., 1979. Lower Cretaceous lithostratigraphy near Galicia Bank. In von Rad, U., Ryan, W.B.F., et al., Init. Repts. DSDP, 47 (Pt. 2): Washington (U.S. Govt. Printing Office), 683-717.

Benson, R.N., 1972. Radiolaria, Leg 12, Deep Sea Drilling Project. In Laughton, A.S., and Berggren, W.A., et al., Init. Repts. DSDP, 12: Washington (U.S. Govt. Printing Office), 1085-1113.

Bjorklund, K.R., and Goll, R.M., 1979. Internal skeletal structures of Collosphaera and Trisolenia: a case of repetitive evolution in the Collosphaeridae (Radiolaria). J. Paleontol., 53:1293-1326.

Blome, C.D., 1992. Radiolarians from Leg 122, Exmouth and Wombat Plateaus, Indian Ocean. In von Rad, U., Haq, B.U., et al., Proc. ODP, Sci. Results, 122: College Station, TX (Ocean Drilling Program), 633-652.

Boltovskoy, D., and Vrba, A., 1988. Classification and geographic distribution of Stylodictya-type radiolarians. Micropaleontology, 34:332-340.

1989. Latitude-related shell patterns in Radiolaria: Botryostrobus auritus/australis morphotypes in the Equatorial to Antarctic Pacific. Mar. Micropaleontol., 13:309-323.

Bütschli, O., 1882. Beiträge zur Kenntnis der Radiolarienskelette, insbesondere der der Cyrtida. Z. Wiss. Zool., 36:485-540.

Campbell, A.S., 1954. Radiolaria. In Moore, R.C. (Ed.), Treatise on Invertebrate Paleontology, Part D, Protista 3. Geol. Soc. Am. and Univ. of Kansas Press, D11-D195.

Campbell, A.S., and Clark, B.L., 1944. Miocene radiolarian faunas from Southern California. Spec. Pap.-Geol. Soc. Am., 51:1-76.

Caulet, J.-P., and Nigrini, C., 1988. The genus Pterocorys (Radiolaria) from the tropical late Neogene of the Indian and Pacific Oceans. Micropaleontology, 34:217-235.

Chen, P.H., 1975. Antarctic Radiolaria. In Hayes, D.E., Frakes, L.A., et al., Init. Repts. DSDP, 28: Washington (U.S. Govt. Printing Office), 437-513.

Cheng, Y.-N., 1986. Taxonomic studies on Upper Paleozoic Radiolaria. Nat. Mus. Natur. Sci. Taiwan, 1:1-311.

Cheng, Y-N., and Yeh, K.-Y., 1989. Radiolaria in surface sediments from west central Pacific near Taiwan (I). Bull. Nat. Mus. Natur. Sci., 1:177-211.

Cita, M.B., 1964. Ricerche micropaleontologiche e stratigraphiche sui sedimenti pelagici del Giuressico superiore e del Cretaceo inferiore nella catena del Monte Baldo. Riv. Ital. Paleontol. Stratigr. Mem., 10:1-182.

Clark, B.L., and Campbell, A.S., 1942. Eocene radiolarian faunas from the Mt. Diablo area, California. Spec. Pap.-Geol. Soc. Am., 39:1-112.

Deflandre, G., 1953. Radiolaires fossiles. In Grassé, P.-P. (Ed.), Traité de Zoologie (Vol. 1): 389-436.

De Wever, P., 1981. Spyrids, artostrobiids, and Cretaceous radiolarians from the western Pacific, Deep Sea Drilling Project Leg 61. In Larson, R.L., and Schlanger, S.O., et al., Init. Repts. DSDP, 61: Washington (U.S. Govt. Printing Office), 507-520.

De Wever, P., and Thiébault, F., 1981. Les radiolaires d'âge Jurassique supérieur à Cretaceé supérieur dans les radiolarites du Pinde-Olonos (Presqu'île de Koroni; Péloponnèse méridional, Grèce). Geobios, 14:577-609.

Dinkelman, M.G., 1973. Radiolarian stratigraphy: Leg 16, Deep Sea Drilling Program. In van Andel, T.H., Heath, G.R., et al., Init. Repts. DSDP, 16: Washington (U.S. Govt. Printing Office), 747-813.

Dreyer, F., 1889. Morphologische Radiolarienstudien. 1. Die Pylombildungen in vergleichend-anatomischer und entwicklungsgeschich tlicher Beziehung bei Radiolarien und bei Protisten überhaupt, nebst System und Beschreibung neuer und der bis jetzt bekannten pylomatischen Spumellarien. Jena. Z. Naturwiss., 23:1-138.

, 1913. Die Polycystinen der Plankton-Expedition. Ergebn. Plankton-Exped. Humboldt-Stiftung. Bd. 3, L. d and e:1-104.

\footnotetext{
Abbreviations for names of organizations and publications in ODP reference lists follow the style given in Chemical Abstracts Service Source Index (published by American Chemical Society).
} 
Dumitrica, P., 1975. Cenomanian Radiolaria at Podul Dimbovitei (Excursion B). In Micropaleontological Guide to the Mesozoic and Tertiary of the Romanian Carpathians. 14th Eur. Micropaleontol. Colloq., 87-89.

Dziewonski, A., Wilkens, R., Firth, J., et al., 1992. Proc. ODP, Init. Repts., 136: College Station, TX (Ocean Drilling Program).

Ehrenberg, C.G., 1847a. Über eine halibiolithische, von Herrn R. Schomburgk entdeckte, vorherrschend aus mikroskopischen Polycystinen gebildete, Gebirgsmasse von Barbados. K. Preuss. Akad. Wiss. Berlin, Bericht, Jahre 1846:382-385.

1847b. Über die mikroskopischen kieselschaligen Polycystinen als mächtige Gebirgsmasse von Barbados und über das Verhältniss der aus mehr als 300 Neuen Arten bestehenden ganz eigenthümlichen Formengruppe jener Felsmasse zu den jetzt lebenden Thieren und zur Kreidebildung. Eine neue Anregung zur Erforschung des Erdlebens. K. Preuss. Akad. Wiss. Berlin, Bericht, Jahre 1846:40-60.

-1854. Mikrogeologie: Das Erden und Felsen Schaffende Wirken des Unsichtbar Kleines Selbständigen Lebens auf der Erde: Leipzig (Leopold Voss).

, 1873. Grössere Felsproben des Polycystinen-Mergels von Barbados mit weiteren Erläuterungen. K. Preuss. Akad. Wiss. Berlin, Monatsbericht, Jahre 1873:213-263.

1875. Fortsetzung der mikrogeologischen Studien als GesammtUebersicht der mikroskopischen Paläontologie gleichartig analysirter $\mathrm{Ge}-$ birgsarten der Erde, mit specieller Rücksicht auf den Polycystinen-Mergel von Barbados. Abh. K. Akad. Wiss. Berlin, Jahre 1875:1-225.

Foreman, H.P., 1973. Radiolaria of Leg 10 with systematics and ranges for the families Amphipyndacidae, Artostrobiidae, and Theoperidae. In Worzel, J. L., Bryant, W., et al., Init. Repts. DSDP, 10: Washington (U.S. Govt. Printing Office), $407-474$.

, 1975. Radiolarians from the North Pacific, Deep Sea Drilling Project, Leg 32. In Larson, R.L., Moberly, R., et al., Init. Repts. DSDP, 32 : Washington (U.S. Govt. Printing Office), 579-676.

Goll, R.M., 1968. Classification and phylogeny of Cenozoic Trissocyclidae (Radiolaria) in the Pacific and Caribbean basins. Part I. J. Paleontol., 42:1409-1432.

1969. Classification and phylogeny of Cenozoic Trissocyclidae (Radiolaria) in the Pacific and Caribbean basins. Part II. J. Paleontol., 43:322-339.

- 1976. Morphological intergradation between modern populations of Lophospyris and Phormospyris (Trissocyclidae, Radiolaria). Micropaleontology, 22:379-418.

1980. Pliocene-Pleistocene radiolaria from the East Pacific Rise and the Galapagos spreading center, Deep Sea Drilling Project Leg 54. In Rosendahl, B.R., Hekinian, R., et al., Init. Repts. DSDP, 54: Washington (U.S. Govt. Printing Office), 425-454.

Haeckel, E., 1862. Die Radiolarien (Rhizopoda Radiolaria): Berlin (Reimer).

1881. Entwurf eines Radiolarien-Systems auf Grund von Studien der Challenger-Radiolarien, Jena. Z. Med. Naturwiss., 15 (Vol. 8, Pt. 3): $418-472$.

1887. Report on the Radiolaria collected by H.M.S. Challenger during the years 1873-1876. Rep. Sci. Results Voy. H.M.S. Challenger. Zool., 18:1-1803.

Hays, J.D., 1965. Radiolaria and late Tertiary and Quaternary history of Antarctic seas. In Llano, G.A. (Ed.), Biology of the Antarctic Seas II. Am. Geophys. Union, Antarct. Res. Ser., 5:125-184.

1970. Stratigraphy and evolutionary trends of Radiolaria in North Pacific deep sea sediments. In Hays, J.D. (Ed.), Geological Investigations of the North Pacific. Mem.-Geol. Soc. Am., 126:185-218.

Hays, J.D., and Berggren, W.A., 1971. Quaternary boundaries and correlations. In Funnell, B.M., and Riedel, W.R. (Eds.), The Micropaleontology of Oceans: Cambridge (Cambridge Univ. Press), 669-691.

Holdsworth, B.K., 1975. Cenozoic radiolaria biostratigraphy: Leg 30: Tropical and Equatorial Pacific. In Andrews, J.E., Packham, G., et al., Init. Repts. DSDP, 30: Washington (U.S. Govt. Printing Office), 499-537.

Johnson, D.A., 1974. Radiolaria from the eastern Indian Ocean, DSDP Leg 22. In von der Borch, C.C., Sclater, J.G., et al., Init. Repts. DSDP, 22: Washington (U.S. Govt. Printing Office), 521-575.

1976. Cenozoic radiolarians from the central Pacific, DSDP Leg 33. In Schlanger, S.O., Jackson, E.D., et al., Init. Repts. DSDP, 33: Washington (U.S. Govt. Printing Office), 425-437.

1978. Cenozoic Radiolaria from the eastern tropical Atlantic. DSDP Leg 41. In Lancelot, Y., Seibold, E., et al., Init. Repts. DSDP, 41 Washington (U.S. Govt. Printing Office), 763-789.
Johnson, D.A., and Knoll, A.H., 1975. Absolute ages of Quaternary radiolarian datum levels in the equatorial Pacific. Quat. Res., 5:99-110.

Johnson, D.A., and Parker, F.L., 1972. Tertiary radiolaria and foraminifera from the equatorial Pacific. Micropaleontology, 18:129-143.

Johnson, D.A., Schneider, D.A., Nigrini, C.A., Caulet, J.-P., and Kent, D.V., 1989. Pliocene-Pleistocene radiolarian events and magnetostratigraphic calibrations for the tropical Indian Ocean. Mar. Micropaleontol., 14:33-66.

Kito, N., 1987. Stratigraphic relation between greenstones and clastic sedimentary rocks in the Kamuikotan Belt, Hokkaido, Japan. Chishitsugaku Zasshi [J. Geol. Soc. Jpn.], 93:21-35.

Kling, S.A., 1973. Radiolaria from the eastern North Pacific, Deep Sea Drilling Project Leg 18. In Kulm, L.D., von Huene, R., et al., Init. Repts. DSDP, 18: Washington (U.S. Govt. Printing Office), 617-671.

Knoll, A.H., and Johnson, D.A., 1975. Late Pleistocene evolution of the collosphaerid radiolarian Buccinosphaera invaginata Haeckel. Micropaleontology, 21:60-68.

Kozlova, G.E., and Gorbovets, A.N., 1966. Radiolyarii verkhnemelovykh i verkhneeotsenovykh otlozhenii Zapadno-Sibirskoi Nizmennosti. Tr. Vses. Neft. Nauchno-Issled. Geologorazved. Inst., 248:1-159.

Ling. H.Y., 1975. Radiolaria: Leg 31 of the Deep Sea Drilling Project. In Karig, D.E., Ingle, J.C., Jr., et al., Init. Repts. DSDP, 31: Washington (U.S. Govt. Printing Office), 703-761.

Ling, H.Y., and Anikouchine, W.A., 1967. Some spumellarian Radiolaria from the Java, Philippine, and Mariana Trenches. J. Paleontol., 41:1481-1491.

Lombari, G., and Lazarus, D.B., 1988. Neogene cycladophorid radiolarians from North Atlantic, Antarctic, and North Pacific deep-sea sediments. Micropaleontology, 34:97-135.

Lozynyak, P.Yu., 1975. Nekotorye radiolyarii melovykh otlozhenii Skibovoi zony Ukrainskikh Karpat. Paleontol. Sborn., Izdatelskoe Obedinenie "Vischa Shkola" Lvokskom Godudarstvennom Univ., 12:48-53.

Matsuyama, H., Kumon, F., and Nakajo, K., 1982. Cretaceous radiolarian fossils from the Hidakagawa Group in the Shimanto Belt, Kii Peninsula, Southwest Japan. In Nakaseko, K. (Ed.), Proc. Ist Japan. Radiolarian Symp., News Osaka Micropaleontol., Spec. Vol., 5:371-382.

Mizutani, S., Nishiyama, H., and Ito, T., 1982. Radiolarian biostratigraphic study of the Shimanto Group in the Nanto-Nansei area, Mie Prefecture, Kii Peninsula, Central Japan. J. Earth Sci., Nagoya Univ., 30:31-107.

Moore, T.C., 1971. Radiolaria. In Tracey, J.I., Jr., Sutton, G.H., et al., Init. Repts. DSDP, 8: Washington (U.S. Govt. Printing Office), 727-775.

1972. Mid-Tertiary evolution of the radiolarian genus Calocycletta. Micropaleontology, 18:144-152.

1973. Radiolaria from Leg 17 of the Deep Sea Drilling Project. In Winterer, E.L., Ewing, J.I., et al., Init. Repts. DSDP, 17: Washington (U.S. Govt. Printing Office), 797-869.

Morley, J.J., and Shackleton, N.J., 1978. Extension of the radiolarian Stylatractus universus as a biostratigraphic datum to the Atlantic Ocean. Geology, 6:309-311.

Nakaseko, K., and Nishimura, A., 1981. Upper Jurassic and Cretaceous Radiolaria from the Shimanto Group in Southwest Japan. Sci. Rep., Coll. Gen. Educ., Osaka Univ, 30:133-203.

Nakaseko, K., Nishimura, A., and Sugano, K., 1979. Cretaceous Radiolaria in the Shimanto Belt, Japan. News Osaka Micropaleontol., Spec. Vol., 2:1-49.

Nakazawa, K., Kumon, F., Kimura, K., Matsuyama, H., and Nakajo, K., 1983. Environment of deposition of Cretaceous chert from the Shimanto Belt, Kii Peninsula, Southwest Japan. In Iijima, A., Hein, J.R., and Siever, R. (Eds.), Siliceous Deposits in the Pacific Region: Amsterdam (Elsevier), 395-412.

Nigrini, C., 1967. Radiolaria in pelagic sediments from the Indian and Atlantic Oceans. Bull. Scripps Inst. Oceanogr., 11:1-125.

1968. Radiolaria from eastern tropical Pacific sediments. Micropaleontology, 14:51-63.

1970. Radiolarian assemblages in the North Pacific and their application to a study of Quaternary sediments in core V20-130. In Hays, J.D. (Ed.), Geological Investigations of the North Pacific. Mem.-Geol. Soc. Am., 126:139-183.

, 1971. Radiolarian zonation in the Quaternary of the equatorial Pacific Ocean. In Funnell, B.M., and Riedel, W.R. (Eds.), The Micropaleontology of Oceans: Cambridge (Cambridge Univ. Press), 443-461.

, 1974. Cenozoic Radiolaria from the Arabian Sea, DSDP Leg 23. In Davies, T.A., Luyendyk, B.P., et al., Init. Repts. DSDP, 26: Washington (U.S. Govt. Printing Office), 1051-1121.

1977. Tropical Cenozoic Artostrobiidae (Radiolaria). Micropa leontology; 23:241-269. 
Nigrini, C., and Lombari, G., 1984. A Guide to Miocene Radiolaria. Spec. Publ. Cushman Found. Foraminiferal Res., 22:S1-S102, N1-N206.

Nigrini, C., and Moore, T.C., Jr., 1979. A Guide to Modern Radiolaria. Spec. Publ. Cushman Found. Foraminiferal Res., 16:S1-S142, N1-N106.

Nishimura, A., 1986. Paleocene radiolarians at Site 384, DSDP. In Recent Progress of Research on Radiolarians and Radiolarian Terranes of Japan. News Osaka Micropaleontol., Spec. Vol., 7:87-93.

Okamura, M., 1980. Radiolarian fossils from the northern Shimanto Belt (Cretaceous) in Kochi Prefecture, Shikoku. Geology and Paleontology of the Shimanto Belt: (Rijnyakosaikai Press), 153-178.

Pessagno, E.A., Jr., 1969. Mesozoic planktonic Foraminifera and Radiolaria. In Ewing, M., Worzel, J.L., et al., Init. Repts. DSDP, 1: Washington (U.S. Govt. Printing Office), 607-621.

- 1976. Radiolarian zonation and stratigraphy of the Upper Cretaceous portion of the Great Valley Sequence, California Coast Ranges, Micropaleontology, Spec. Publ., 2:1-95.

1977. Lower Cretaceous radiolarian biostratigraphy of the Great Valley Sequence and Franciscan Complex, California Coast Ranges. Spec. Publ. Cushman Found. Foraminiferal Res., 15:1-87.

Pessagno, E.A., Jr., Blome, C.D., and Longoria, J.F., 1984. A revised radiolarian zonation for the Upper Jurassic of western North America. Bull. Am. Paleontol., 87:1-51.

Pessagno, E.A., Jr., and Michael, F.Y., 1974. Results of shore laboratory studies on Mesozoic planktonic foraminifera from Leg 26 Sites 255, 256, 257, and 258. In Barker, P., Dalziel, I.W.D., et al., Init. Repts. DSDP, 36: Washington (U.S. Govt. Printing Office), 969-972.

Pessagno, E.A., Jr., and Newport, R.L., 1972. A technique for extracting Radiolaria from radiolarian cherts. Micropaleontology, 18:231-234.

Pessagno, E.A., Jr., Whalen, P.A., and Yeh, K., 1986. Jurassic Nassellariina (Radiolaria) from North American geological terranes. Bull. Am. Paleontol., 91:1-68.

Petrushevskaya, M.G., 1971. Radiolyarii Nassellaria v planktone Mirovogo Okeana (Radiolarians of the Ocean). Issled. Fauny Morei, 9:1-294.

1975. Cenozoic radiolarians of the Antarctic, Leg 29, DSDP. In Kennett, J.P., Houtz, R.E., et al., Init. Repts. DSDP, 29: Washington (U.S. Govt. Printing Office), 541-675.

Petrushevskaya, M.G., and Kozlova, G.E., 1972. Radiolaria, Leg 14, Deep Sea Drilling Project. In Hayes, D.E., Pimm, A.C., et al., Init. Repts. DSDP, 14: Washington (U.S. Govt. Printing Office), 495-648.

Popofsky, A., 1913. Die Nassellarien des Warmwassergebietes. Dtsch. Sudpolar-Exped., 1901-1903, 14 (Zool. Vol. 6):217-416.

Renz, G.W., 1976. The distribution and ecology of Radiolaria in the Central Pacific plankton and surface sediments. Bull. Scripps Inst. Oceanogr, 22:1-267.

, 1990. Late Ordovician (Caradocian) radiolarians from Nevada. Micropaleontology, 36:367-377.

Riedel, W.R., 1967. Subclass Radiolaria. In Harland, W.B., Holland, C.H., House, M.R., Hughes, N.F., Reynolds, A.B., Rudwick, M.J.S., Satterthwaite, G.E., Tarlo, L.B.H., and Willey, E.C. (Eds.), The Fossil Record. Geol. Soc. London, 291-298.

, 1971. Systematic classification of polycystine Radiolaria. In Funnel, B.M., and Riedel, W.R. (Eds.), The Micropaleontology of Oceans. Cambridge (Cambridge Univ. Press), 649-661.

Riedel, W.R., and Funnell, B.M., 1964. Tertiary sediment cores and microfossils from the Pacific Ocean floor. Q. J. Geol. Soc. London, 120:305-368.

Riedel, W.R., and Hays, J.D., 1969. Cenozoic Radiolaria from Leg 1. In Ewing, M., and Worzel, J.L., et al., Init. Repts. DSDP, 1: Washington (U.S. Govt. Printing Office), $400-402$.

Riedel, W.R., and Sanfilippo, A., 1970. Radiolaria, Leg 4, Deep Sea Drilling Project. In Bader, R.G., Gerard, R.D., et al., Init. Repts. DSDP, 4: Washington (U.S. Govt. Printing Office), 503-575.

1971. Cenozoic Radiolaria from the western tropical Pacific, Leg 7. In Winterer, E.L., Riedel, W.R., et al., Init. Repts. DSDP, 7 (Pt. 2): Washington (U.S. Govt. Printing Office), 1529-1672.

, 1974. Radiolaria from the southern Indian Ocean, DSDP Leg 26. In Davies, T.A., Luyendyk, B.P., et al., Init. Repts DSDP, 26: Washington (U.S. Govt. Printing Office), 771-813.

1978. Stratigraphy and evolution of tropical Cenozoic radiolarians. Micropaleontology, 24:61-96.

Sanfilippo, A., and Riedel, W.R., 1973. Cenozoic Radiolaria (exclusive of theoperids, artostrobids and amphipyndacids) from the Gulf of Mexico,
Deep Sea Drilling Project Leg 10. In Worzel, J.L., Bryant, W., et al., Init. Repts. DSDP, 10: Washington (U.S. Govt. Printing Office), 475-611.

, 1980. A revised generic and suprageneric classification of the Artiscins (Radiolaria). J. Paleontol., 54:1008-1011.

1982. Revision of the radiolarian genera Theocotyle, Theocotylissa, and Thyrsocyrtis. Micropaleontology, 28:170-188.

1992. The origin and evolution of Pterocorythidae (Radiolaria): a Cenozoic phylogenetic study. Micropaleontology, 38:1-36.

Sanfilippo, A., Westberg-Smith, M.J., and Riedel, W.R., 1985. Cenozoic radiolaria. In Bolli, H.M., Saunders, J.B,, and Perch-Nielsen, K. (Eds.), Plankton Stratigraphy: Cambridge (Cambridge Univ. Press), 631-712.

Schaaf, A., 1981. Late early Cretaceous Radiolaria from Deep Sea Drilling Project Leg 48. In Montadert, L., Roberts, D.G., et al., Init. Repts. DSDP. 48: Washington (U.S. Govt. Printing Office), 419-470.

1984. Les radiolaires du Crétacé inférieur et moyen: biologie et systématique. Sci. Geol. Mem., 75:1-189.

Schmidt-Effing, R., 1980. Radiolarien der mittel-Kreide aus dem Santa ElenaMassiv von Costa Rica. Neues Jahrb. Geol. Palaeontol. Abh., 160:24I-257.

Schreiber, B.C., 1969. New evidence concerning the age of the Hawaiian Ridge. Geol. Soc. Am Bull., 80:2601-2604.

Squinabol, S., 1903. Le Radiolaire dei noduli selciosi nella Scaglia degli Euganei. Contrib. I. Riv. Ital. Paleontol., 9:105-151.

Strelkov, A.A., and Reshetnyak, V.V., 1971. Kolonialnye radiolyarii Spumellaria Mirovogo okeana (Colonial Spumellarian radiolarians of the world ocean). Issled. Fauny Morei, 9:295-418.

Suyari, K., 1986. Restudy of the northern Shimanto Subbelt in eastern Shikoku. J. Sci. Univ. Tokushima, 19:45-54.

Suyari, K., and Hashimoto, H., 1985. A radiolarian assemblage from the Izumi Group of eastern Shikoku. J. Sci. Univ. Tokushima, 18:103-127.

Suyari, K., and Yamasaki, T., 1988. Microfossil age of the northern margin of the Shimanto South Subbelt in Shikoku. J. Sci. Univ. Tokushima, 21:107-133.

Takahashi, K., and Honjo, S., 1981. Vertical flux of Radiolaria: a taxon-quantitative sediment trap study from the western tropical Atlantic. Micropaleontology, 27:140-190.

Takemura, A., 1986. Classification of Jurassic nassellarians (Radiolaria). Palaeontographica Abt. A, 195:29-74.

Takemura, A., and Yamauchi, M., 1984. Cephalic structure of Cornutella (Radiolaria) and its phylogeny. News Osaka Micropaleontol., 12:33-39.

Taketani, Y., 1982. Cretaceous radiolarian biostratigraphy of the Urakawa and Obira areas, Hokkaido. Sci. Rep. Tohoku Univ. Ser. 2, 52:1-75.

Tracey, J.I., Jr., Sutton, G.H., et al., 1971. Init. Repts. DSDP, 8: Washington (U.S. Govt. Printing Office).

Tumanda, F.P., 1989. Cretaceous radiolarian biostratigraphy in the Esashi Mountain area, Northern Hokkaido, Japan. Sci. Rep., Univ. Tsukuba, Sect. $B, 10: 1-44$.

Vinassa de Regny, P.E., 1900. Radiolari Miocenici Italiani. Mem. R. Acad. Sci. Inst. Bologna, Ser. 5, 8:227-257.

Wetzel, O., 1935. Die Mikropalaeontologie des Heiligenhafener Kieseltones (Ober-Eözan). Niedersaechs. Geol, Verein, Jahresbericht, 27:41-75.

Winterer, E.L., Riedel, W.R., et al., 1971. Init. Repts. DSDP, 7 (Pts. 1 and 2): Washington (U.S. Govt. Printing Office).

Yamauchi, M., 1982. Upper Cretaceous radiolarians from Northern Shimanto Belt along the course of Shimanto River, Kochi Prefecture, Japan. News Osaka Micropaleontol., 5:383-397.

1986. The distribution of radiolarian assemblages in surface sediments from the northwestern Pacific. In Recent Progress of Research on Radiolarians and Radiolarian Terranes in Japan. News Osaka Micropaleontol. Spec. Vol., 7:141-156.

Yao, A., 1984. Subdivision of the Mesozoic complex in Kii-Yura area, southwest Japan and its bearing on the Mesozoic basin development in the southern Chichibu Terrane. J. Geosci., Osaka City Univ., 27:41-103.

Zittel, K.A., 1876. Ueber einige fossile Radiolarien auf der norddeutschen Kreide. Z. Dtsch. Geol. Gesell., 28:75-86.

Date of initial receipt: 28 August 1992

Date of acceptance: 5 January 1993

Ms 136SR-201 

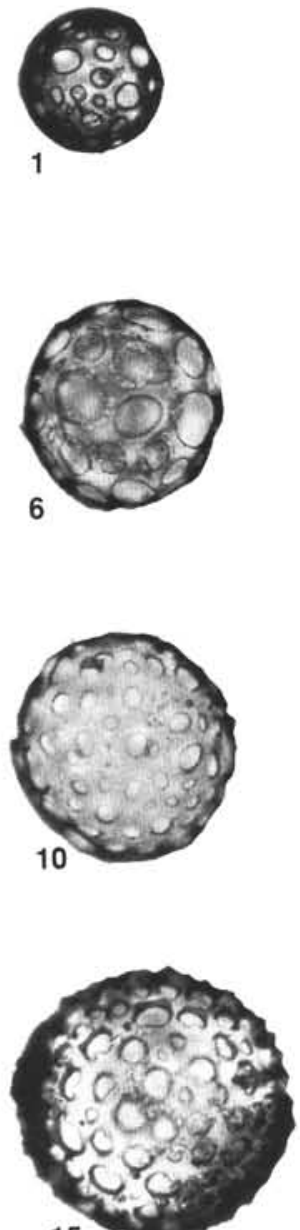

15
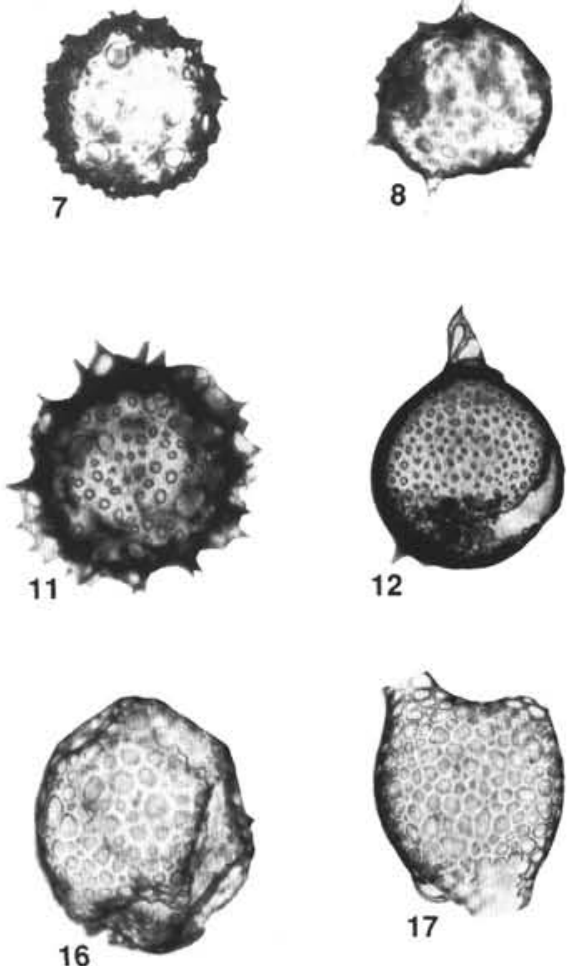

17

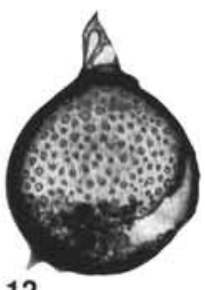

12
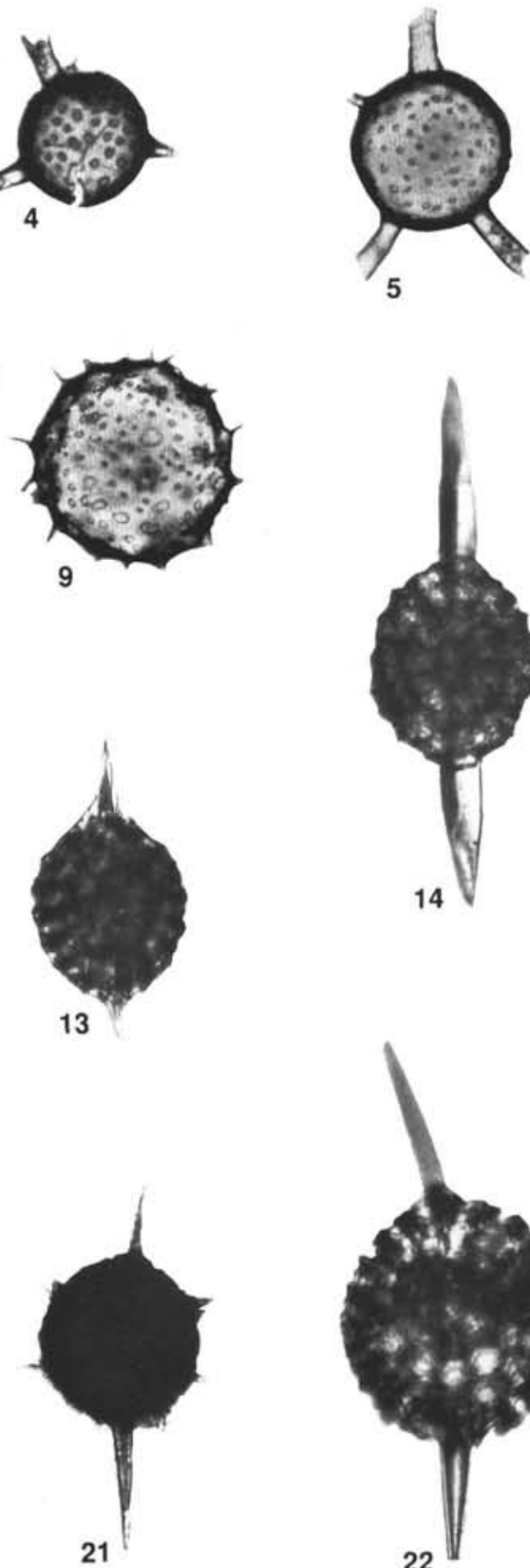
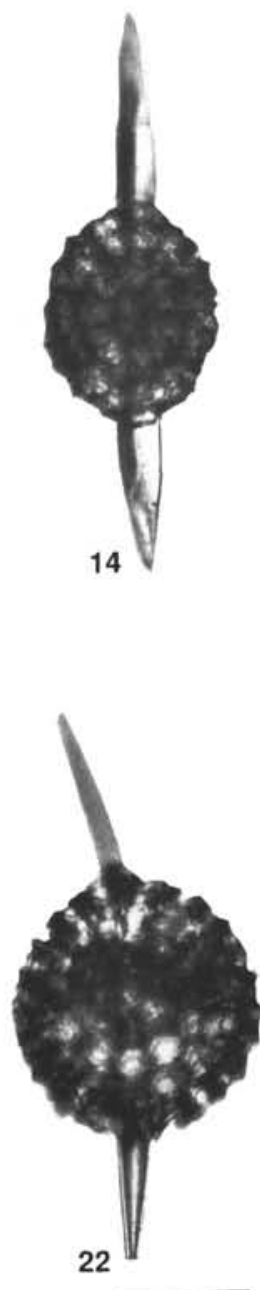

Plate 1. Scale bar $=80 \mu \mathrm{m} . \quad 1,2,10$. Collosphaera spp., Samples $842 \mathrm{~B}-1 \mathrm{H}-2,126-128 \mathrm{~cm}, 843 \mathrm{C}-1 \mathrm{H}-2,19-21 \mathrm{~cm}$, and $843 \mathrm{C}-1 \mathrm{H}-1,34-36 \mathrm{~cm}$, respectively. 3. Siphonosphaera cyathina, Sample $843 \mathrm{C}-1 \mathrm{H}-1,34-36 \mathrm{~cm}$. 4, 5. Siphonosphaera polysiphonia, Samples $843 \mathrm{C}-1 \mathrm{H}-2,129-131 \mathrm{~cm}$, and $843 \mathrm{C}-1 \mathrm{H}-1,34-36 \mathrm{~cm}$, respectively. 6. Collosphaera macrophora, Sample 843C-1H-1, 34-36 cm. 7. Acrosphaera flammabunda, Sample 842B-1H-3, 9-11 cm. 8,9. Acrosphaera spinosa, Sample 843C-1H-2, 19-21 cm. 11. Acrosphaera lapaccea, Sample 843C-1H-1, 34-36 cm. 12. Otosphaera polymorpha, Sample 842B-1H-2, 9-11 $\mathrm{cm}$. 13. Stylatractus sp., Sample $843 \mathrm{C}-1 \mathrm{H}-1,34-36 \mathrm{~cm}$. 14. Stylatractus giganteus, Sample $843 \mathrm{C}-1 \mathrm{H}-1,34-36 \mathrm{~cm}$. 15. Acrosphaera murrayana, Sample 842B-1H-1, 9-11 cm. 16. Collosphaera tuberosa, Sample 843C-1H-1, 34-36 cm. 17. Disolenia sp., Sample 842B-1H-3, 9-11 cm. 18, 19. Collosphaera orthoconus, Sample 843C-1H-1, 34-36 cm. 20. Axoprunum stauraxonium, Sample 842B-1H-3, 9-11 cm. 21. Axoprunum angelinum, Sample 843C-1H-2, $129-131 \mathrm{~cm}$. 22. Stylacontarium acquilonium, Sample 842B-1H-1, 9-11 cm. 

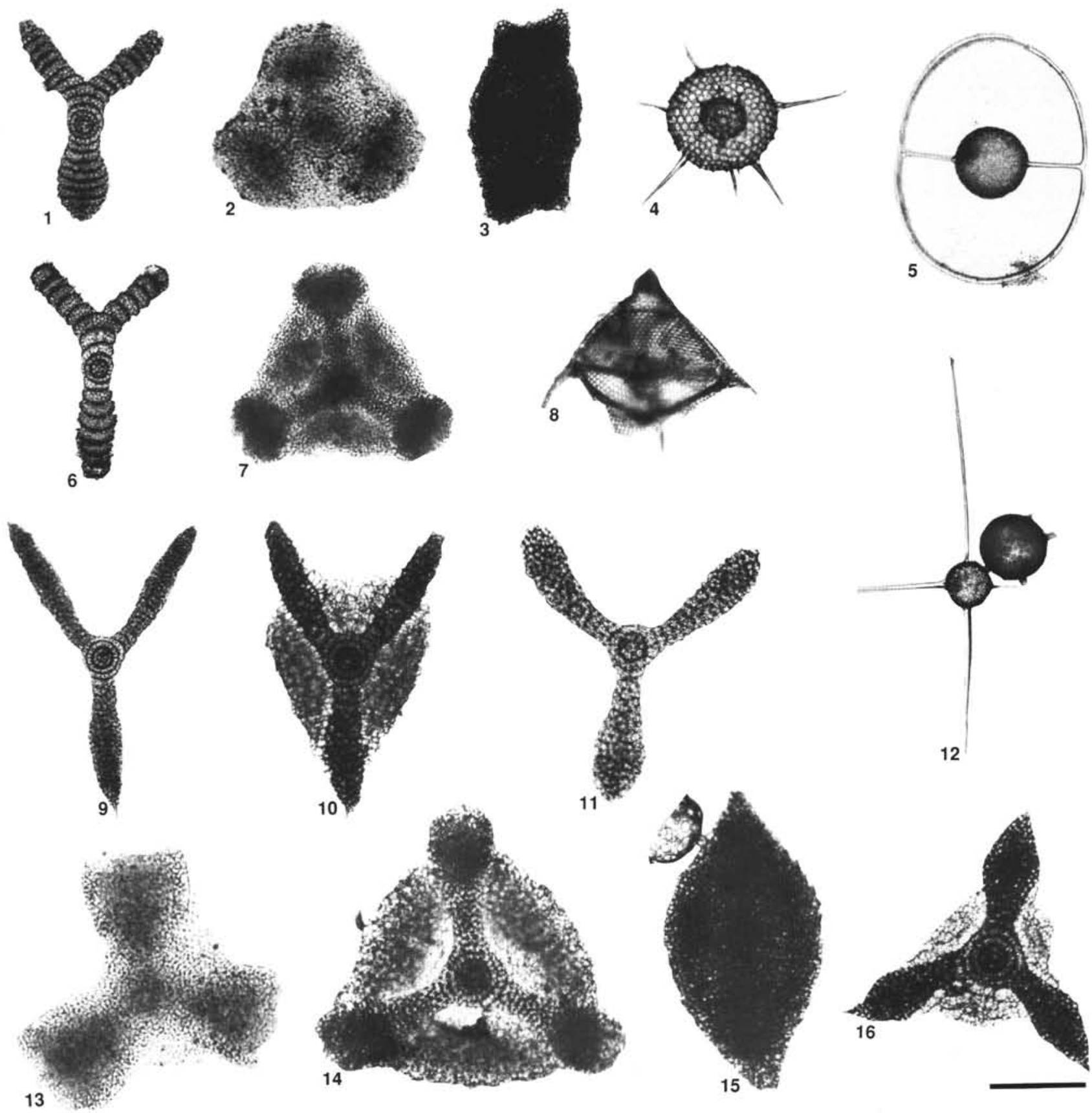

Plate 2. Scale bar $=130 \mu \mathrm{m} . \quad$ 1, 6. Amphirhopalum ypsilon, Samples $842 \mathrm{~B}-1 \mathrm{H}-1,9-11 \mathrm{~cm}$, and $842 \mathrm{~B}-1 \mathrm{H}-1,126-128 \mathrm{~cm}$, respectively. 2. Dictyocoryne truncatum, Sample 842B-1H-1,9-11 cm. 3. Spongobrachium ellipticum, Sample 842B-1H-2, 126-128 cm. 4. Heliodiscus astericus, Sample 842B-1H-1, 9-11 cm. 5. Saturnalis circularis, Sample $843 \mathrm{C}-1 \mathrm{H}-2,19-21 \mathrm{~cm}$. 7. Hymeniastrum sp. aff. H. euclidis, Sample $842 \mathrm{~B}-1 \mathrm{H}-1,126-128 \mathrm{~cm}$. 8. Dictyocodon sp., Sample 843C-1H-2, 129-131 cm. 9, 10. Euchitonia elegans, Samples 842B-1H-1,9-11 cm, and 843C-1H-2, 129-131 cm, respectively. 11. Euchitonia furcata, Sample 842B-1H-1, 9-11 cm. 12. Xiphosphaera tesseractis, Sample 842B-1H-2, 126-128 cm. 13. Dictyocoryne sp. aff. D. truncatum, Sample 842B-1H-1, 9-11 cm. 14. Hymeniastrum euclidis, Sample 842B-1H-1, 9-11 cm. 15. Spongobrachium sp. aff. S. ellipticum, Sample 843C-1H-2, 129-131 cm. 16. Dictyocoryne profunda, Sample 843C-1H-2, 129-131 cm. 

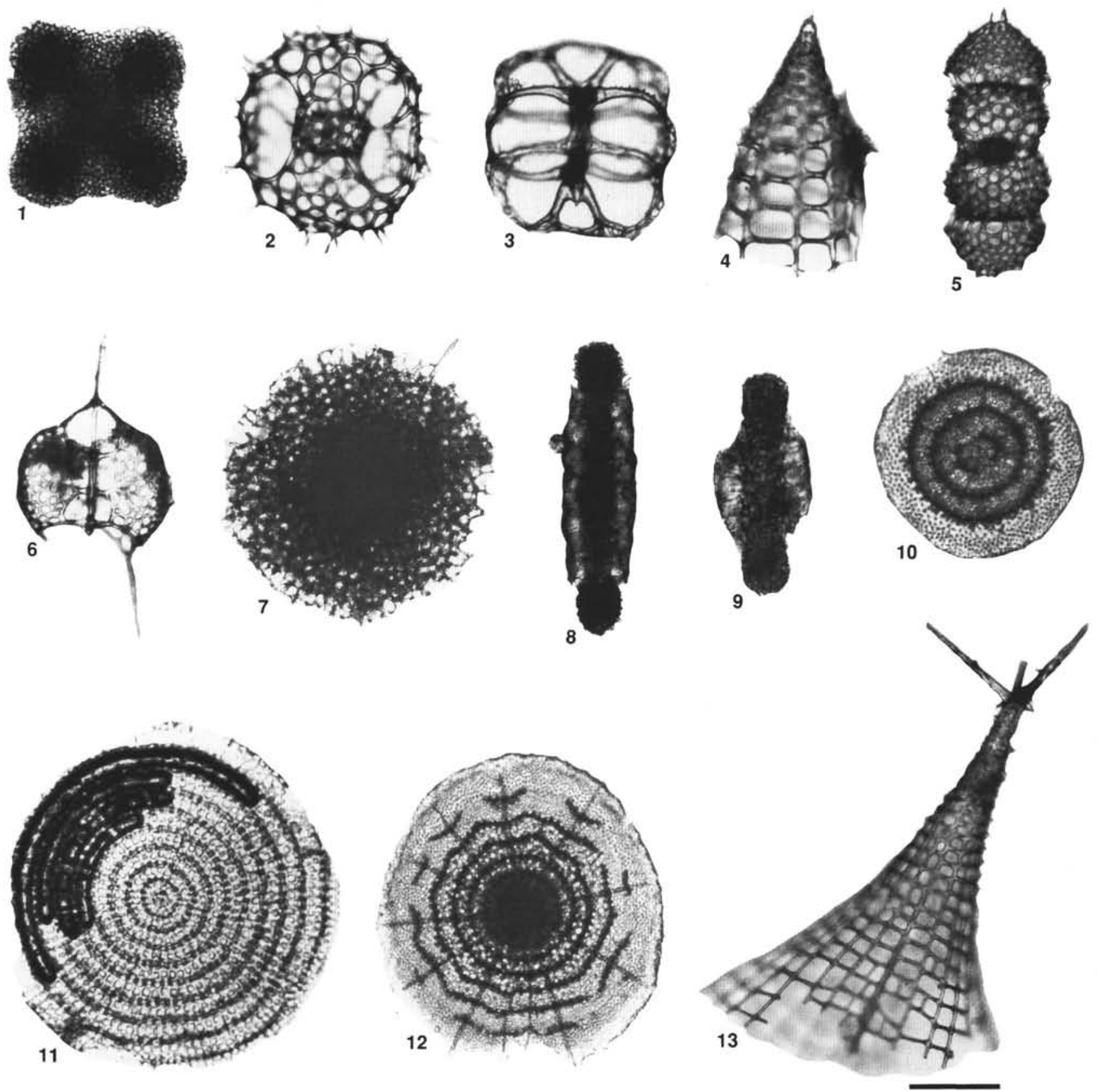

Plate 3. Scale bar $=100 \mu \mathrm{m}$. 1. Spongaster tetras tetras, Sample $842 \mathrm{~B}-1 \mathrm{H}-1,126-128 \mathrm{~cm}$. 2. Tetrapyle octacantha, Sample $843 \mathrm{C}-1 \mathrm{H}-2,19-21 \mathrm{~cm}$. 3. Liriospyris reticulata, Sample $843 \mathrm{C}-1 \mathrm{H}-2,19-21 \mathrm{~cm}$. 4. Peripyramis circumtexta, Sample $843 \mathrm{C}-1 \mathrm{H}-1,34-36 \mathrm{~cm}$. 5. Didymocyrtis tetrathalamus tetrathalamus, Sample 843C-1H-1, 34-36 cm. 6. Tholospyris sp., Sample 842B-1H-3, 126-128 cm. 7. Spongotrochus(?) venustum, Sample 843C-1H-1, 34-36 cm. 8, 9. Spongocore puella, Sample 842B-1H-1, 126-128 cm. 10. Circodiscus microporus, Sample $843 \mathrm{C}-1 \mathrm{H}-2,19-21 \mathrm{~cm}$. 11. Stylodictya validispina, Sample $842 \mathrm{~B}-1 \mathrm{H}-1,9-11 \mathrm{~cm}$. 12. Ommatodiscus murrayi, Sample $843 \mathrm{C}-1 \mathrm{H}-2,129-131 \mathrm{~cm}$. 13. Plectopyramis dodecomma, Sample $842 \mathrm{~B}-1 \mathrm{H}-5,8-10 \mathrm{~cm}$. 

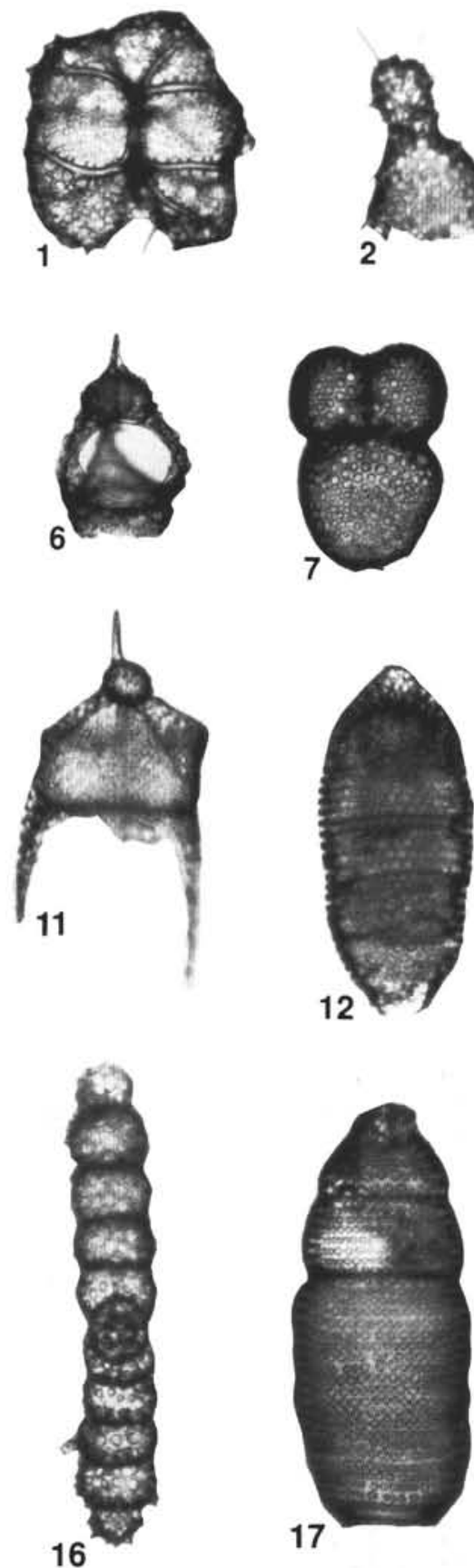
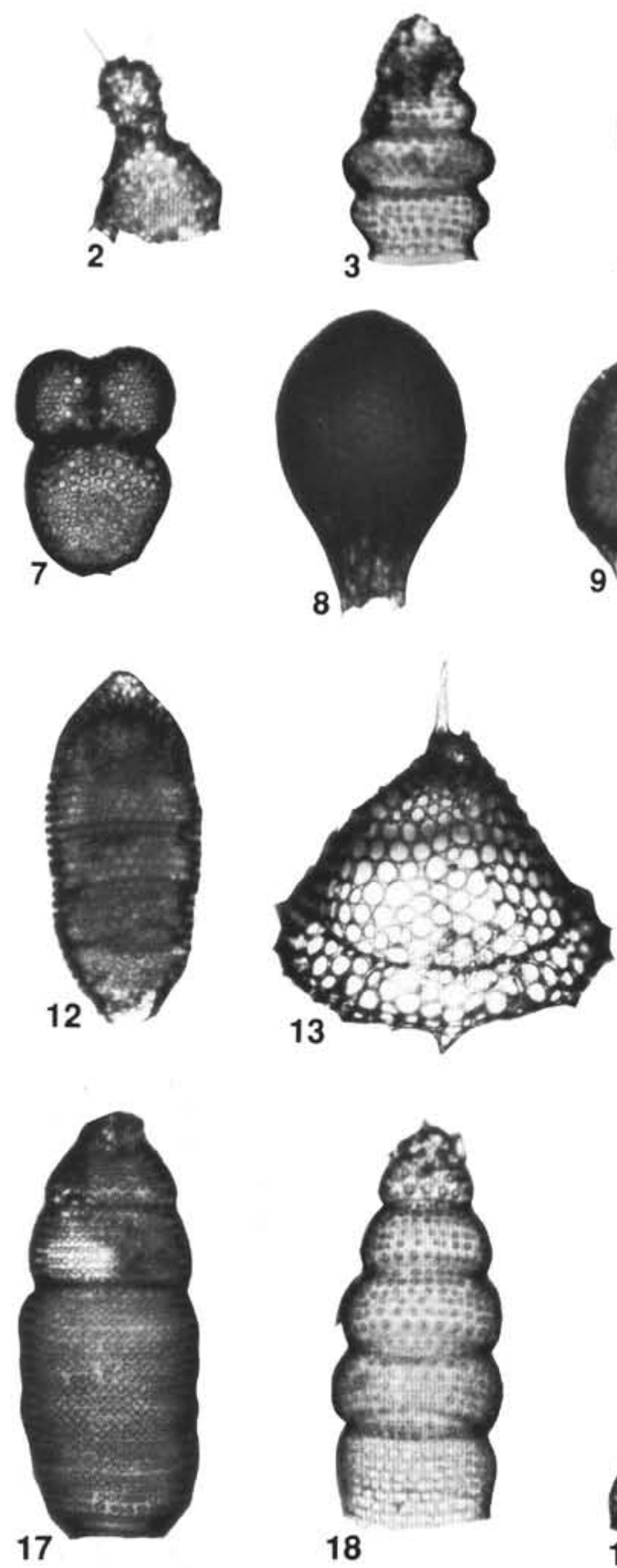
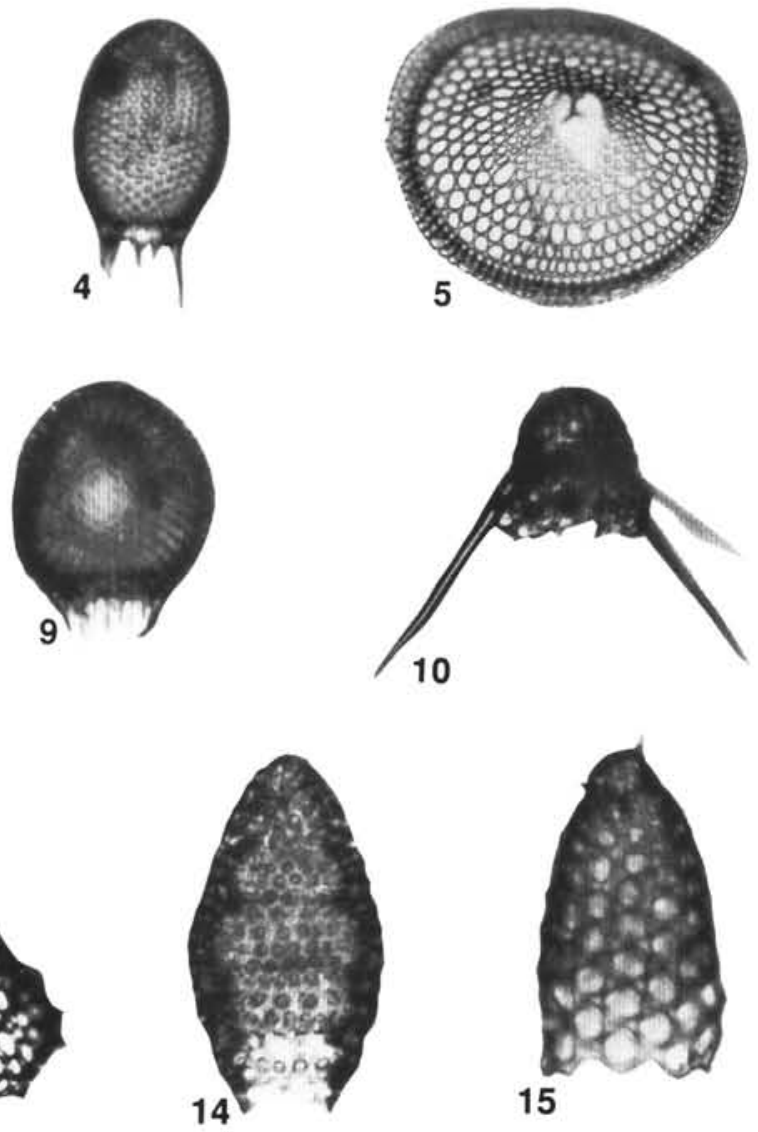

15

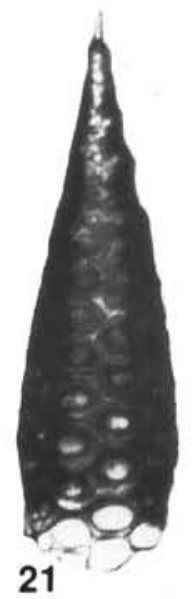

Plate 4. Scale bar $=100 \mu \mathrm{m}$. 1. Liriospyris reticulata, Sample $842 \mathrm{~B}-1 \mathrm{H}-2,126-128 \mathrm{~cm}$. 2. Lophophaena cylindrica, Sample $842 \mathrm{~B}-1 \mathrm{H}-2,126-128 \mathrm{~cm}$. 3. Botryostrobus sp.. Sample 843C-1H-1,34-36 cm. 4, 8,9. Carpocanistrum spp., Sample 842B-1H-5, 8-10 cm. 5. Theopilium tricostatum, Sample 842B-1H-2, $126-128 \mathrm{~cm}$. 6. Clathrocanium sp., Sample $842 \mathrm{~B}-1 \mathrm{H}-2,126-128 \mathrm{~cm}$. 7. Phormospyris stabilis stabilis, Sample $842 \mathrm{~B}-1 \mathrm{H}-1,126-128 \mathrm{~cm} . \quad 10$. Achipilium sp. aff. A. macropus, Sample 842B-1H-2, 126-128 cm. 11. Pterocanium praetextum praetextum, Sample 843C-1H-1, 34-36 cm. 12. Lithocampe sp., Sample 842B-1H-1,9-11 cm. 13. Cycladophora sp. cf. C. cabrilloensis, Sample 842B-1H-3,9-11 cm. 14. Botryostrobus sp. aff. B. aquilonarius, Sample 842B-1H-1, 9-11 cm. 15. Cycladophora(?) sp., Sample $843 \mathrm{C}-1 \mathrm{H}-1,34-36 \mathrm{~cm}$. 16. Tessarastrum straussi, Sample $843 \mathrm{C}-1 \mathrm{H}-1$, 34-36 cm. 17. Phormostichoartus corbula, Sample $843 \mathrm{C}-1 \mathrm{H}-1,34-36 \mathrm{~cm}$. 18. Botryostrobus auritus/australis group, Sample $843 \mathrm{C}-1 \mathrm{H}-1,34-36 \mathrm{~cm}$. 19. Stichopera pectinata group, Sample $843 \mathrm{C}-1 \mathrm{H}-1,34-36 \mathrm{~cm}$. 20, 21. Cornutella profunda, Samples $842 \mathrm{~B}-1 \mathrm{H}-5,8-10 \mathrm{~cm}$, and $843 \mathrm{C}-1 \mathrm{H}-2,129-131 \mathrm{~cm}$, respectively. 

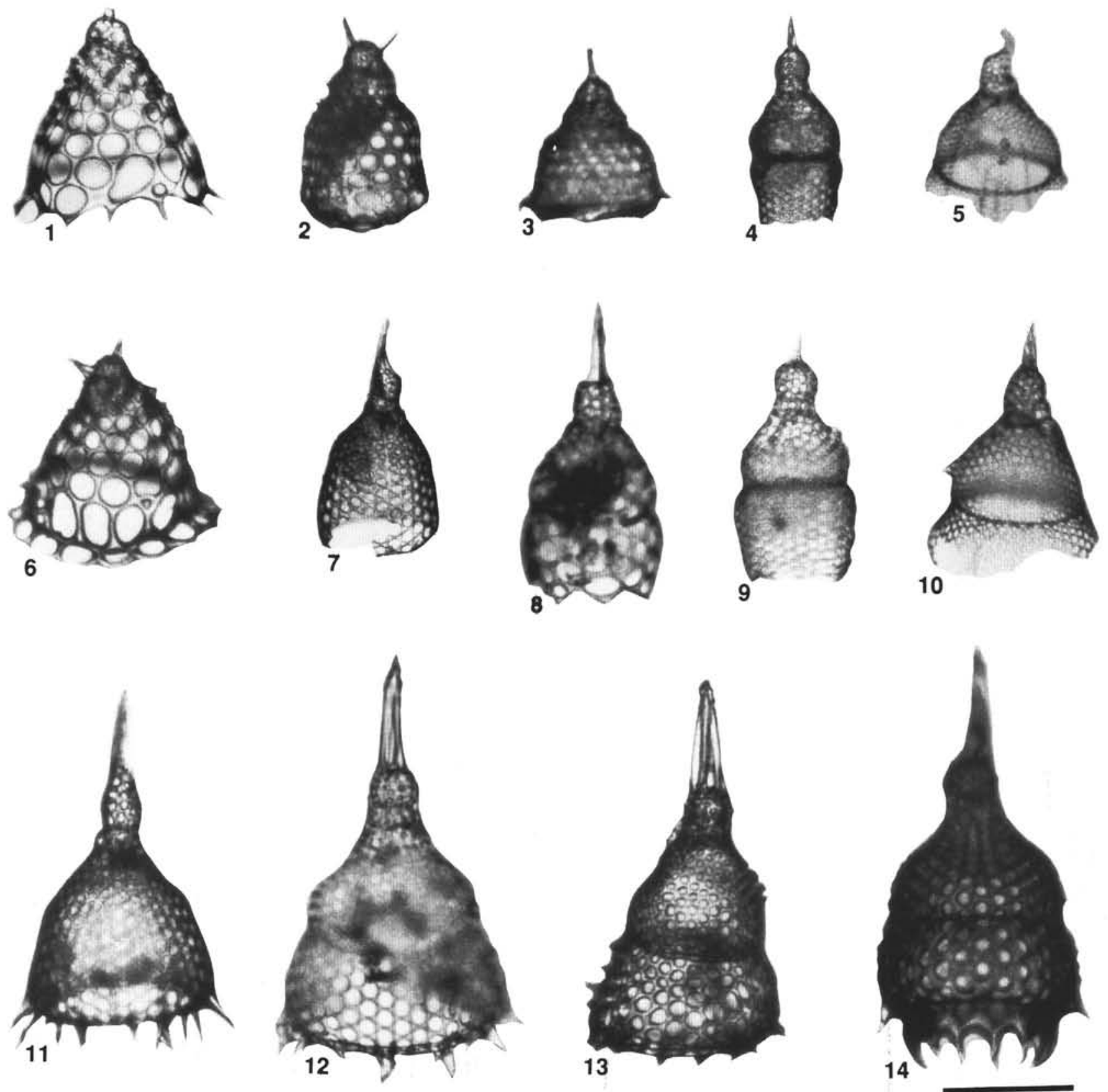

Plate 5. Scale bar $=130 \mu \mathrm{m}$. 1,6. Cycladophora sp. aff. C. conica, Samples $842 \mathrm{~B}-1 \mathrm{H}-1,9-11 \mathrm{~cm}$, and $843 \mathrm{C}-1 \mathrm{H}-1,34-36 \mathrm{~cm}$, respectively. 2,3. Cycladophora spp., Sample 843C-1H-1, 34-36 cm. 4, 9. Theocorythium trachelium sp. aff. T.t. dianae, Samples $842 \mathrm{~B}-1 \mathrm{H}-3,9-11 \mathrm{~cm}$, and $842 \mathrm{~B}-1 \mathrm{H}-1,9-11 \mathrm{~cm}$, respectively. 5. Pterocorys hertwigii, Samples 842B-1H-1, 28-30 cm. 7. Anthocyrtidium sp. aff. A. zanguebaricum, Sample 843C-1H-2, 129-131 cm. 8. Lamprocyrtis(?) hannai, Sample 842B-1H-5, 8-10 cm. 10. Pterocorys sabae (Ehrenberg), Sample 843C-1H-1, 34-36 cm. 11. Anthocyrtidium ophirense, Sample 843C-1H-2, 129-131 cm. 12. Lamprocyclas maritalis polypora, Sample 842B-1H-1,9-11 cm. 13. Lamprocylas maritalis sp. aff. L.m. polypora, Sample 843C-1H-1,34-36 cm. 14. Lamprocyclas maritalis maritalis, Sample $843 \mathrm{C}-1 \mathrm{H}-1,34-36 \mathrm{~cm}$. 

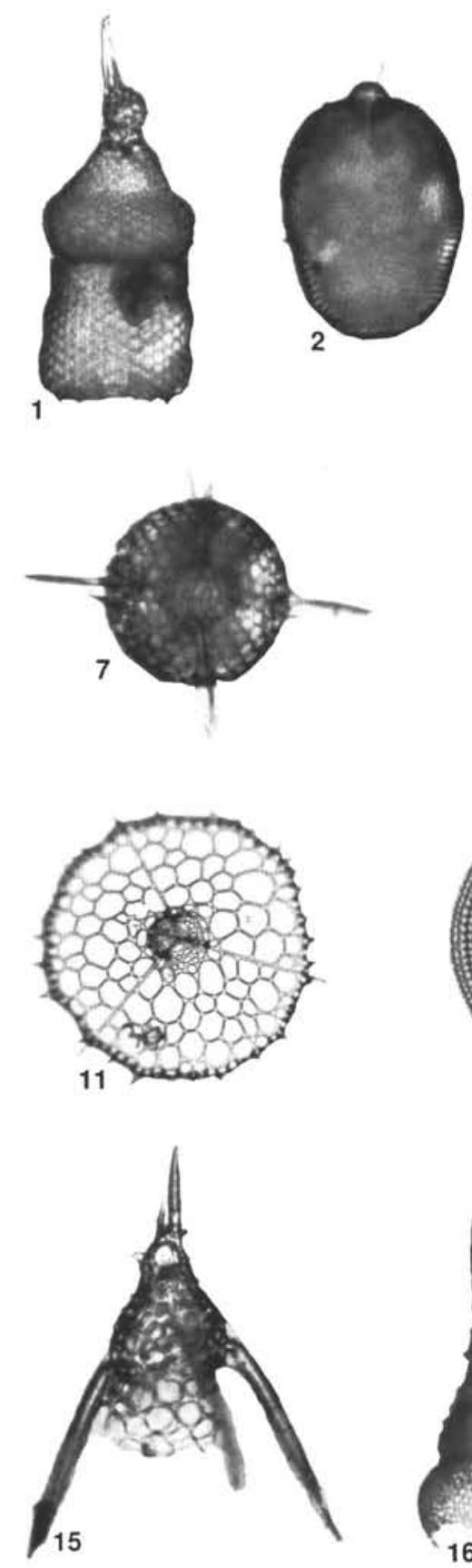
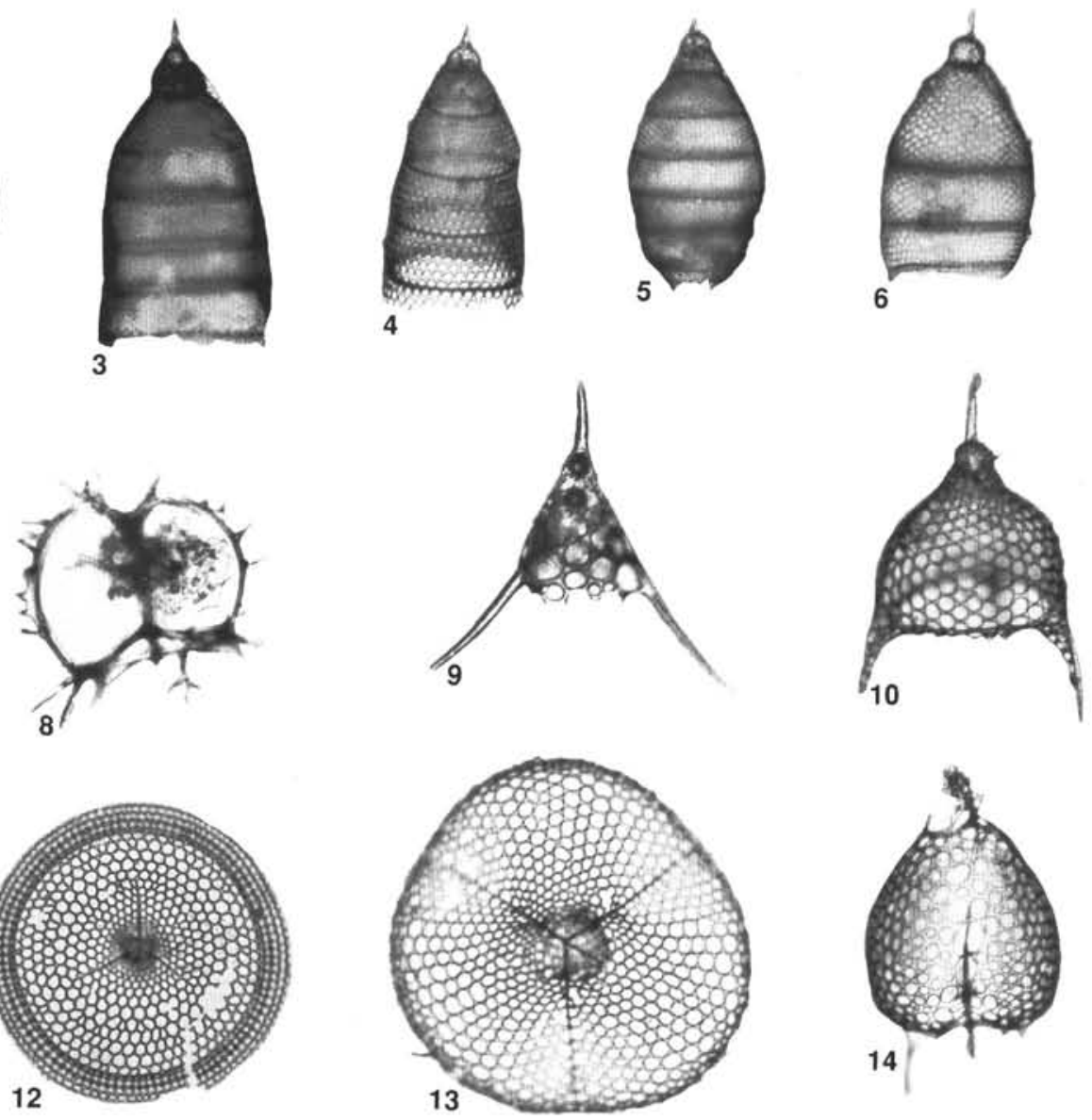

12
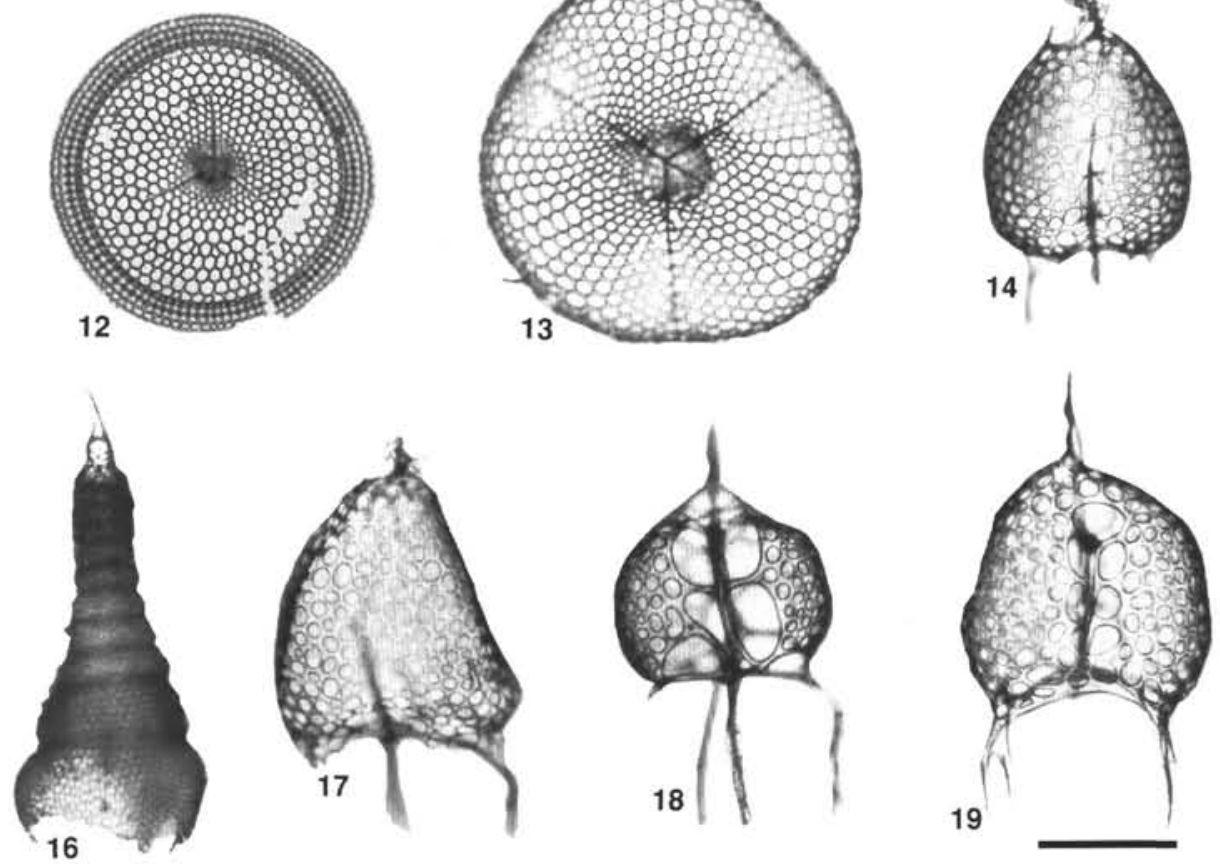

Plate 6. Scale bar $=80 \mu \mathrm{m}$. 1. Theocorythium trachelium trachelium, Sample $843 \mathrm{C}-1 \mathrm{H}-1,34-36 \mathrm{~cm}$. 2. Lithopera bacca, Sample $842 \mathrm{~B}-1 \mathrm{H}-1,9-11 \mathrm{~cm}$. 3. Eucyrtidium hexagonatum. Sample $843 \mathrm{C}-1 \mathrm{H}-1,34-36 \mathrm{~cm}$. 4, 5. Eucyrtidium sp. aff. E. acuminatum, Sample $843 \mathrm{C}-1 \mathrm{H}-1,34-36 \mathrm{~cm}$. 6. Eucyrtidium anomalum, Sample 843C-1H-1, 34-36 cm. 7. Hexacontium enthacanthum, Sample 842B-1H-5, 8-10 cm. 8. Giraffospyris angulata, Sample 843C-1H-2, 129-131. 9, 15. Dictyophimus crisiae. Sample 842B-1H-3,9-11 cm. 10. Pterocanium trilobum, Sample 843C-1H-1, 34-36 cm. 11. Sethophormis sp. aff. S. pentalactis, Sample 842B-1H-1, 126-128 cm. 12. Theopilium tricostatum. Sample 842B-1H-3, 9-11 cm. 13. Sethophormis pentalactis, Sample 843C-1H-2, 129-131 cm. 14, 18, 19. Tholospyris spp. $(14,18=$ Sample $843 \mathrm{C}-1 \mathrm{H}-1,34-36 \mathrm{~cm} ; 19=$ Sample $842 \mathrm{~B}-1 \mathrm{H}-1,9-11 \mathrm{~cm})$. 16. Stichopera pectinata group, Sample $843 \mathrm{C}-1 \mathrm{H}-1,34-36 \mathrm{~cm}$. 17. Tholospyris fornicata, Sample $843 \mathrm{C}-1 \mathrm{H}-1,34-36 \mathrm{~cm}$. 

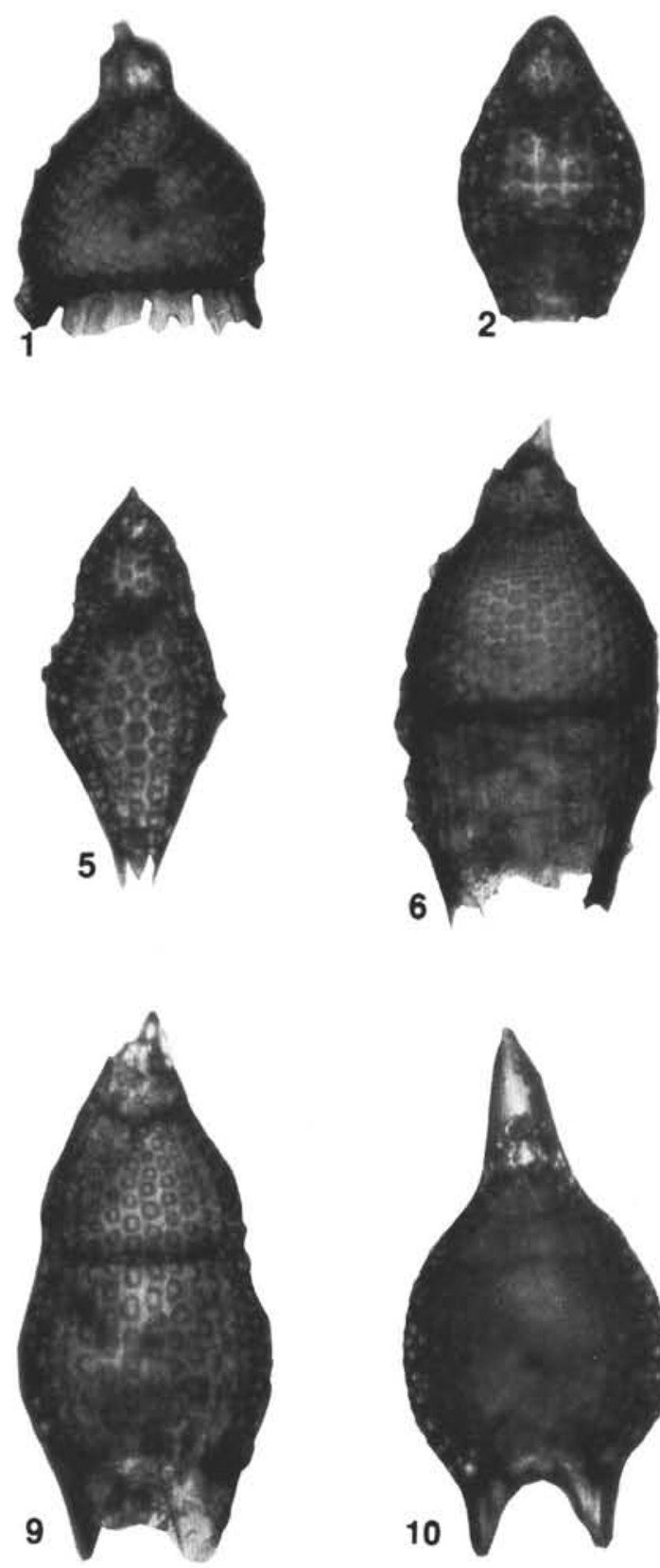
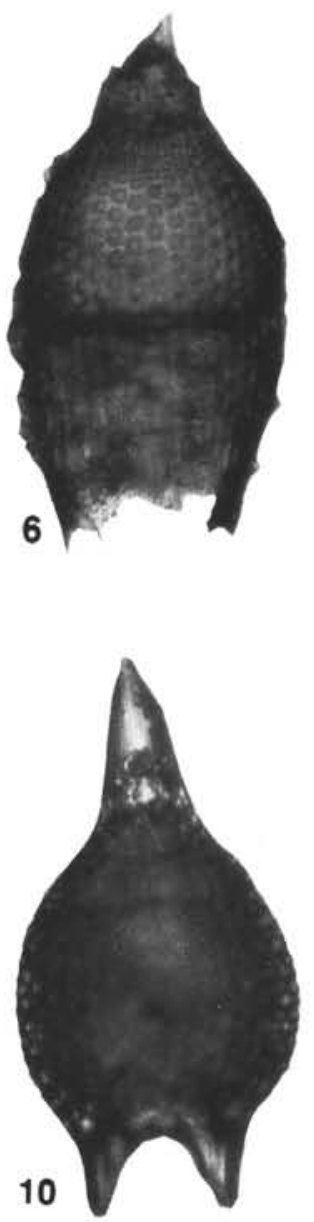
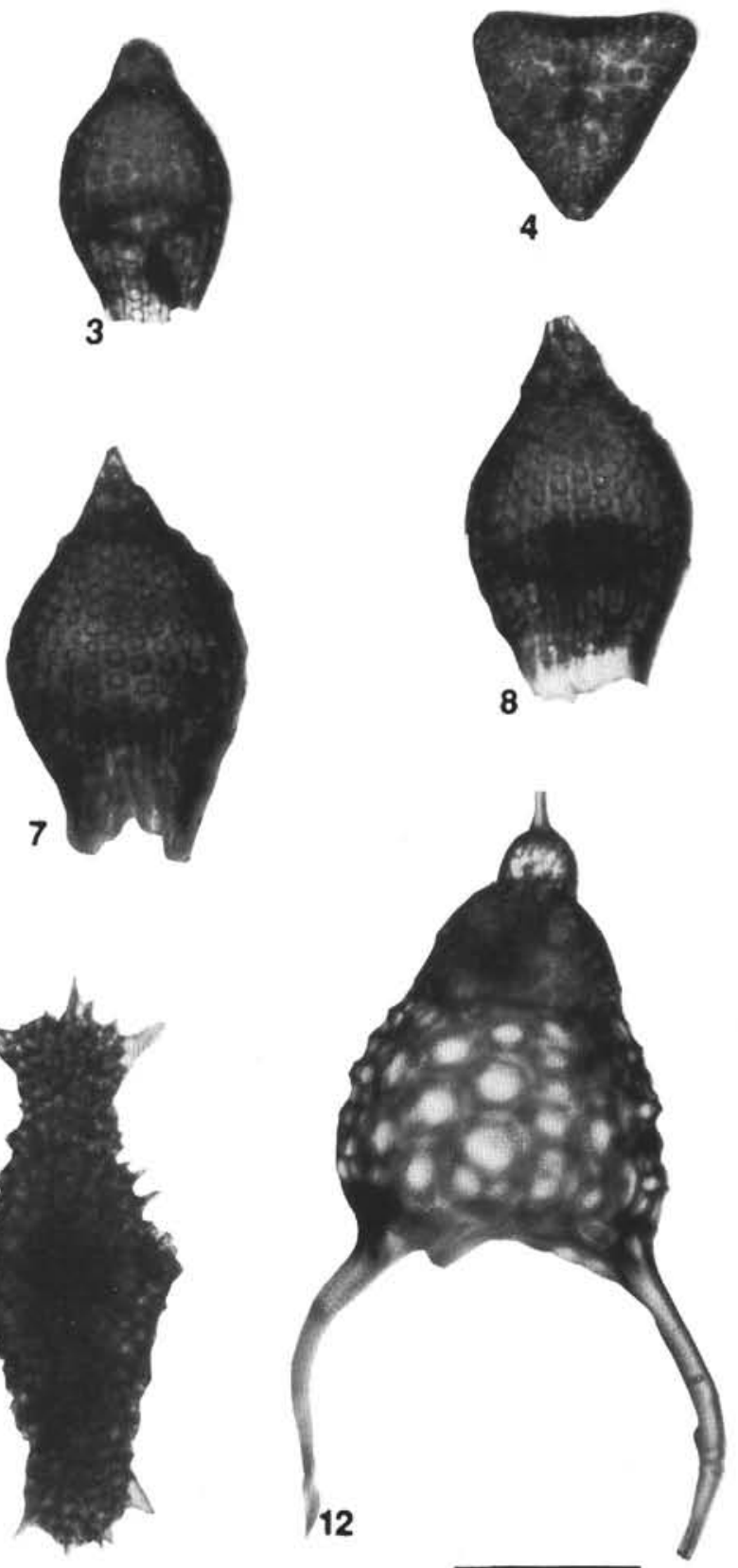

Plate 7. Scale bar $=100 \mu \mathrm{m}$. Reworked early to middle Eocene radiolarians extracted from a volcanic sand layer at 7.5 mbsf (Sample 842A-1H-6, 66-67 cm). 1. Calocyclas hispida. 2. Buryella tetradica. 3. Cryptocarpium sp. 4. Giraffospyris lata. 5. Phormocyrtis striata striata. 6. Podocyrtis (Lampterium) sp. aff. P. (L.) acalles. 7, 8. Podocyrtis (Podocyrtis) papalis. 9. Podocyrtis (Lampterium) sinuosa. 10. Theocotylissa alpha. 11. Stylotrochus quadribrachiatus quadribrachiatus. 12. Thyrsocyrtis (Pentalacorys) triacantha. 


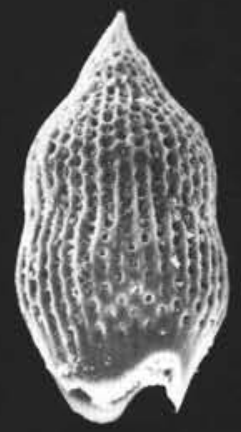

1

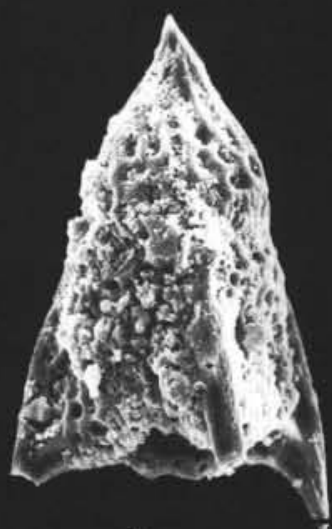

5

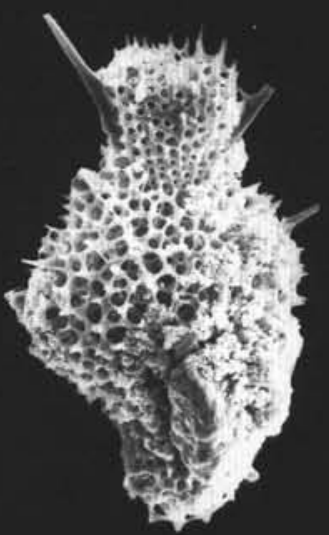

9

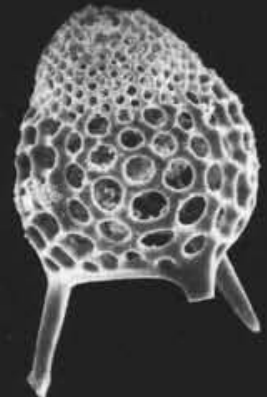

2

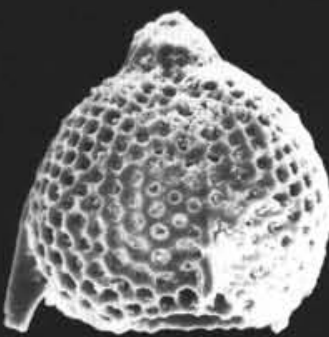

6

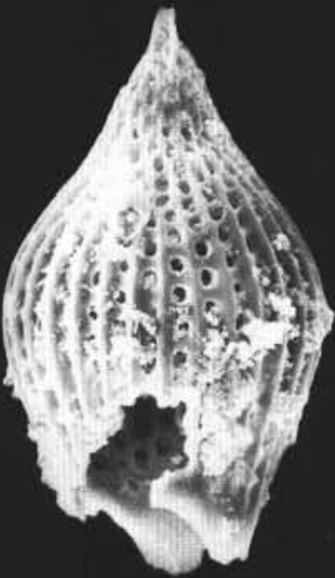

10
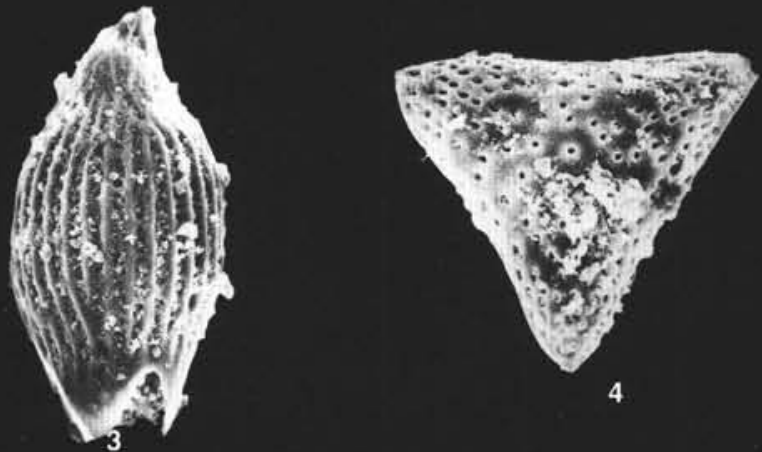

4
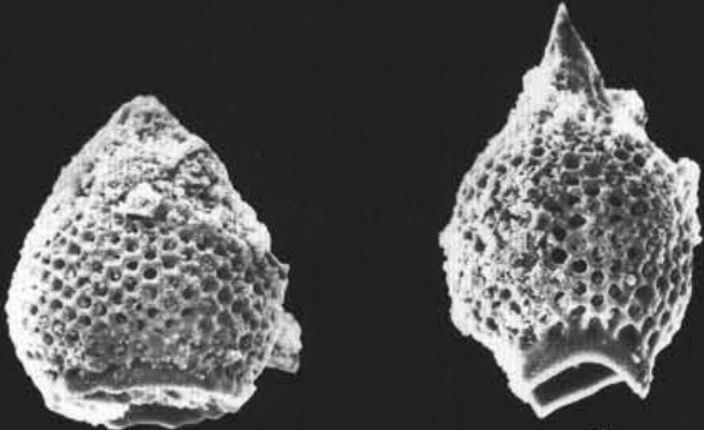

8

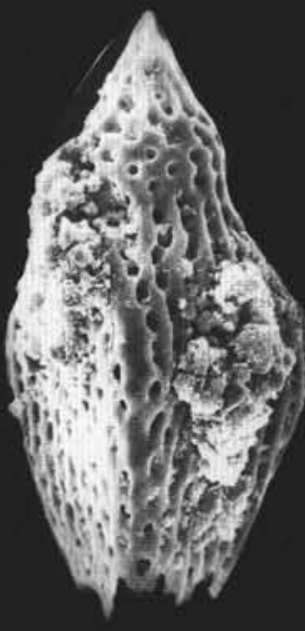

12

Plate 8. SEM photomicrographs of reworked Eocene radiolarians from Hole 842A. Scale bar lengths given in parentheses. 1. Podocyrtis (Lampterium) acalles, Sample 842A-1H-6, 124-126 cm $(60 \mu \mathrm{m})$. 2. Thyrsocyrtis (Pentalacorys) triacantha, Sample 842A-1H-6, 124-126 cm ( $85.7 \mu \mathrm{m})$. 3. Podocyrtis (Podocyrtis) sp. aff. $P$. $(P$.$) papalis, Sample 842A-1H-7, 6-8 cm (85.7 \mu \mathrm{m})$. 4. Giraffospyris lata, Sample $842 \mathrm{~A}-1 \mathrm{H}-6,124-126 \mathrm{~cm}(60 \mu \mathrm{m})$. 5. Rhopalocanium sp., Sample $842 \mathrm{~A}-1 \mathrm{H}-6,66-67 \mathrm{~cm}(85.7 \mu \mathrm{m})$. 6. Lychnocanoma sp., Sample $842 \mathrm{~A}-1 \mathrm{H}-6,66-67 \mathrm{~cm}(85.7 \mu \mathrm{m})$. 7. Theocotylissa ficus, Sample $842 \mathrm{~A}-1 \mathrm{H}-7,6-8 \mathrm{~cm}(85.7$ $\mu \mathrm{m})$. 8, 11. Theocotylissa alpha, Sample $842 \mathrm{~A}-1 \mathrm{H}-6,66-67 \mathrm{~cm}(85.7 \mu \mathrm{m}, 60 \mu \mathrm{m})$. 9. Stylotrochus quadribrachiatus quadribrachiatus, Sample $842 \mathrm{~A}-1 \mathrm{H}-6$, $66-67 \mathrm{~cm}(85.7 \mu \mathrm{m}) . \quad 10$. Podocyrtis (Podocyrtis) papalis, Sample $842 \mathrm{~A}-1 \mathrm{H}-6,66-67 \mathrm{~cm}(60 \mu \mathrm{m}) . \quad 12$. Phormocyrtis striata exquisita, Sample $842 \mathrm{~A}-1 \mathrm{H}-6$, $66-67 \mathrm{~cm}(60 \mu \mathrm{m})$. 


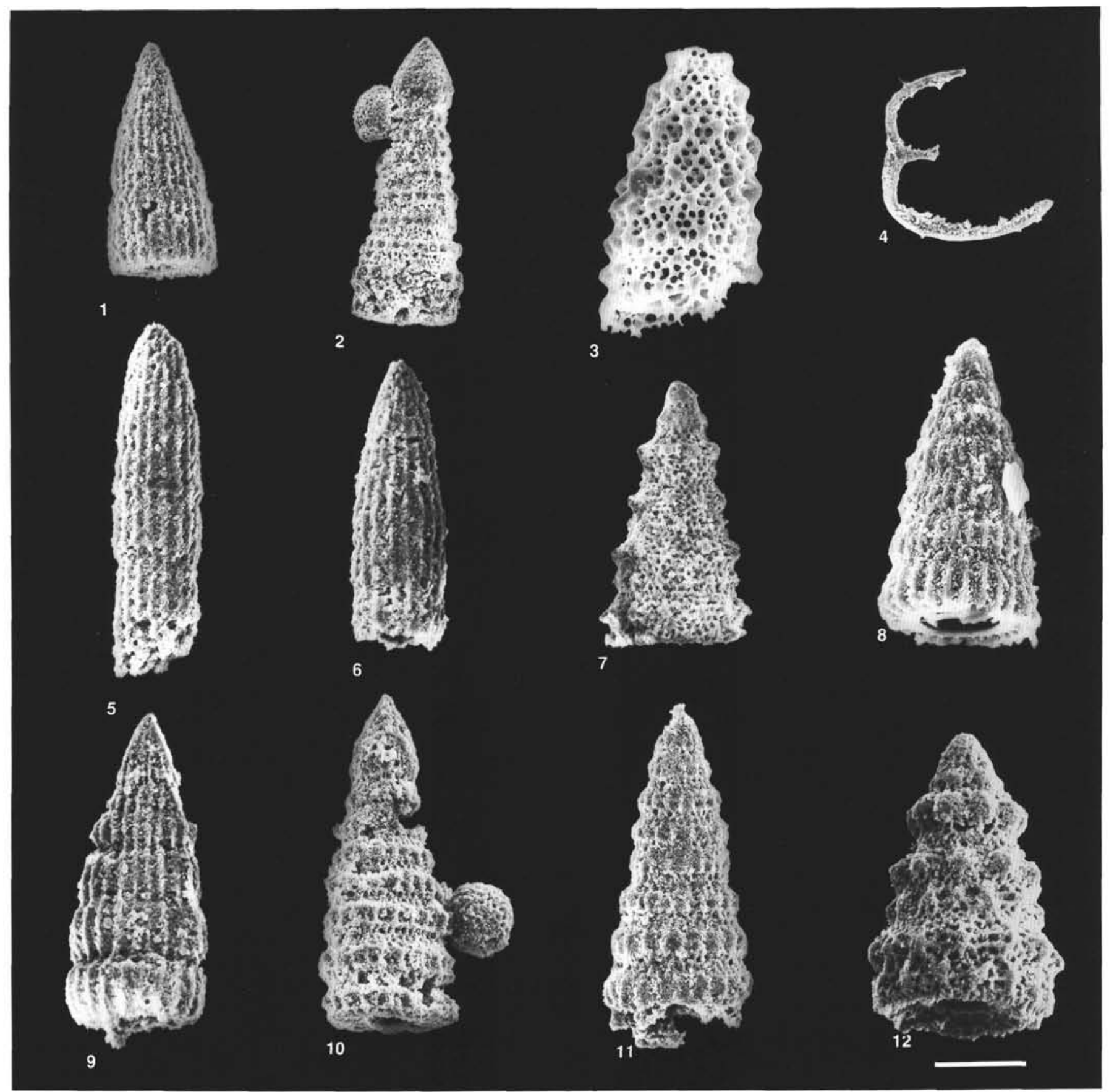

Plate 9. SEM photomicrographs of Cretaceous radiolarians from cherts, Site 842. Scale bar lengths given in parentheses. 1. Archaeodictyomitra sp., Sample $842 \mathrm{C}-1 \mathrm{~W}-1,65-67 \mathrm{~cm}(85.7 \mu \mathrm{m}) . \quad$ 2, 10. Pseudodictyomitra pseudomacrocephala, Sample $842 \mathrm{C}-1 \mathrm{~W}-1,65-67 \mathrm{~cm}(150 \mu \mathrm{m}, 85.7 \mu \mathrm{m}) .3$. 3itus(?) sp.., wash core between 842B-8X and 842B-9X $(85.7 \mu \mathrm{m})$. 4. Acanthocircus sp., Sample $842 \mathrm{C}-1 \mathrm{~W}-1,65-67 \mathrm{~cm}(60 \mu \mathrm{m})$. 5. Archaeodictyomitra sp. aff. A. sliteri, Sample 842C-1W-1. 65-67 cm $(85.7 \mu \mathrm{m})$. 6. Archaeodictyomitra sliteri, Sample 842C-1W-1, 65-67 cm $(85.7 \mu \mathrm{m})$. 7. Novixitus sp., Sample 842C-IW-1, 65-67 cm $(85.7 \mu \mathrm{m}) . \quad 8$, 9. Dictyomitra sp. cf. D. napaensis $(8=$ wash core between $842 \mathrm{~B}-8 \mathrm{X}$ and $842 \mathrm{~B}-9 \mathrm{X}[60 \mu \mathrm{m}, 60 \mu \mathrm{m}] ; 9=\mathrm{Sample} 842 \mathrm{C}-1 \mathrm{~W}-1,65-67 \mathrm{~cm}$ [85.7 $\mu \mathrm{m}])$. 11. Pseudodictyomitra sp. aff. P. pentacolaensis, Sample $842 \mathrm{C}-1 \mathrm{~W}-1,65-67 \mathrm{~cm}(85.7 \mu \mathrm{m}) . \quad 12$. Novixitus mclaughlini, Sample $842 \mathrm{C}-1 \mathrm{~W}-1,65-67 \mathrm{~cm}$ $(85.7 \mu \mathrm{m})$. 